\title{
Measurement of Inclusive $\pi^{0}$ Production in hadronic $\mathrm{Z}^{0}$ Decays
}

\author{
DELPHI Collaboration
}

\begin{abstract}
An analysis is presented of inclusive $\pi^{0}$ production in $Z^{0}$ decays measured with the DELPHI detector. At low energies, $\pi^{0}$ decays are reconstructed by using pairs of converted photons and combinations of converted photons and photons reconstructed in the barrel electromagnetic calorimeter (HPC). At high energies (up to $x_{p}=2 \cdot p_{\pi} / \sqrt{s}=0.75$ ) the excellent granularity of the HPC is exploited to search for two-photon substructures in single showers. The inclusive differential cross section is measured as a function of energy for $q \bar{q}$ and $b \bar{b}$ events. The number of $\pi^{0}$ s per hadronic $Z^{0}$ event is $N\left(\pi^{0}\right) / Z_{\text {had }}^{0}=$ $9.2 \pm 0.2$ (stat) \pm 1.0 (syst) and for $b \bar{b}$ events the number of $\pi^{0}$ 's is $\mathrm{N}\left(\pi^{0}\right) / b \bar{b}=$ $10.1 \pm 0.4$ (stat) \pm 1.1 (syst). The ratio of the number of $\pi^{0}$ 's in $b \bar{b}$ events to hadronic $Z^{0}$ events is less affected by the systematic errors and is found to be $1.09 \pm 0.05 \pm 0.01$. The measured $\pi^{0}$ cross sections are compared with the predictions of different parton shower models. For hadronic events, the peak position in the $\xi_{p}=\ln \left(1 / x_{p}\right)$ distribution is $\xi_{p}^{\star}=3.90_{-0.14}^{+0.24}$. The average number of $\pi^{0}$ 's from the decay of primary $\mathrm{B}$ hadrons is found to be $\mathrm{N}(B \rightarrow$ $\left.\pi^{0} X\right) /$ B hadron $=2.78 \pm 0.15$ (stat) \pm 0.60 (syst) .
\end{abstract}


W.Adam $^{50}$, T.Adye ${ }^{37}$, E.Agasi ${ }^{31}$, I.Ajinenko ${ }^{42}$, R.Aleksan ${ }^{39}$, G.D.Alekseev ${ }^{16}$, P.P.Allport ${ }^{22}$, S.Almehed ${ }^{24}$, S.J.Alvsvaag ${ }^{4}$, U.Amaldi ${ }^{9}, \quad$ S.Amato $^{47}, \quad$ A.Andreazza $^{28}$, M.L.Andrieux ${ }^{14}$, P.Antilogus ${ }^{9}, \quad$ W-D.Apel ${ }^{17}$, Y.Arnoud ${ }^{39}$, B. Asman ${ }^{44}$, J-E.Augustin ${ }^{19}$, A.Augustinus ${ }^{31}$, P.Baillon ${ }^{9}$, P.Bambade ${ }^{19}$, F.Barao ${ }^{21}$, R.Barate $^{14}$, G.Barbiellini $^{46}$, D.Y.Bardin ${ }^{16}$, G.J.Barker ${ }^{35}$, A.Baroncelli ${ }^{40}$, O.Barring ${ }^{24}$, J.A.Barrio ${ }^{26}$, W.Bartl ${ }^{50}$, M.J.Bates ${ }^{37}$, M.Battaglia ${ }^{15}$, M.Baubillier ${ }^{23}$, J.Baudot ${ }^{39}$, K-H.Becks ${ }^{52}$, M.Begalli ${ }^{6}, \quad$ P.Beilliere $^{8}$, Yu.Belokopytov $^{9}$, K.Belous ${ }^{42}$, A.C.Benvenuti ${ }^{5}$, M.Berggren ${ }^{47}$, D.Bertrand ${ }^{2}$, F.Bianchi ${ }^{45}$, M.Bigi ${ }^{45}$, M.S.Bilenky ${ }^{16}$, P.Billoir ${ }^{23}$, D.Bloch ${ }^{10}$, M.Blume ${ }^{52}$, S.Blyth ${ }^{35}$, V.Bocci ${ }^{38}$, T.Bolognese ${ }^{39}$, M.Bonesini ${ }^{28}$, W.Bonivento ${ }^{28}$, P.S.L.Booth ${ }^{22}$, G.Borisov ${ }^{42}$, C.Bosio ${ }^{40}$, S.Bosworth ${ }^{35}$, O.Botner ${ }^{48}$, B.Bouquet $^{19}$, C.Bourdarios ${ }^{9}$, T.J.V.Bowcock ${ }^{22}$, M.Bozzo ${ }^{13}$, P.Branchini ${ }^{40}$, K.D.Brand ${ }^{36}$, T.Brenke ${ }^{52}$, R.A.Brenner ${ }^{15}$, C.Bricman ${ }^{2}$, L.Brillault ${ }^{23}$, R.C.A.Brown ${ }^{9}$, P.Bruckman ${ }^{18}$, J-M.Brunet ${ }^{8}$, L.Bugge ${ }^{33}$, T.Buran ${ }^{33}$, T.Burgsmueller ${ }^{52}$, P.Buschmann ${ }^{52}$, A.Buys ${ }^{9}$, M.Caccia $^{28}$, M.Calvi ${ }^{28}$, A.J.Camacho Rozas ${ }^{41}$, T.Camporesi $^{9}$, V.Canale ${ }^{38}$, M.Canepa ${ }^{13}$, K.Cankocak ${ }^{44}$, F.Cao $^{2}, \quad$ F.Carena ${ }^{9}$, P.Carrilho ${ }^{47}$, L.Carroll ${ }^{22}$, C.Caso ${ }^{13}$, M.V.Castillo Gimenez ${ }^{49}$, A.Cattai ${ }^{9}$, F.R.Cavallo ${ }^{5}$, L.Cerrito ${ }^{38}$, V.Chabaud ${ }^{9}$, Ph.Charpentier ${ }^{9}$, L.Chaussard $^{25}$, J.Chauveau $^{23}$, P.Checchia ${ }^{36}$, G.A.Chelkov ${ }^{16}$, R.Chierici ${ }^{45}$, P.Chliapnikov ${ }^{42}$, P.Chochula ${ }^{7}$, V.Chorowicz ${ }^{9}$, V.Cindro ${ }^{43}$, P.Collins ${ }^{9}$, J.L.Contreras ${ }^{19}$, R.Contri ${ }^{13}$, E.Cortina ${ }^{49}$, G.Cosme ${ }^{19}$, F.Cossutti ${ }^{46}$, H.B.Crawley ${ }^{1}$, D.Crennell $^{37}$, G.Crosetti ${ }^{13}$, J.Cuevas Maestro ${ }^{34}$, S.Czellar ${ }^{15}$, E.Dahl-Jensen ${ }^{29}$, J.Dahm ${ }^{52}$, B.Dalmagne ${ }^{19}$, M.Dam $^{29}$, G.Damgaard ${ }^{29}$, P.D.Dauncey ${ }^{37}$, M.Davenport ${ }^{9}$, W.Da Silva ${ }^{23}$, C.Defoix ${ }^{8}$, A.Deghorain ${ }^{2}$, G.Della Ricca ${ }^{46}$, P.Delpierre ${ }^{27}$, N.Demaria ${ }^{35}$, A.De Angelis ${ }^{9}$, H.De Boeck ${ }^{2}$, W.De Boer ${ }^{17}$, S.De Brabandere ${ }^{2}$, C.De Clercq $^{2}$, C.De La Vaissiere ${ }^{23}$, B.De Lotto ${ }^{46}$, A.De Min ${ }^{36}$, L.De Paula ${ }^{47}$, C.De Saint-Jean ${ }^{39}$, H.Dijkstra ${ }^{9}$, L.Di Ciaccio ${ }^{38}$, F.Djama ${ }^{10}$, J.Dolbeau ${ }^{8}$, M.Donszelmann ${ }^{9}$, K.Doroba ${ }^{51}$, M.Dracos ${ }^{10}$, J.Drees ${ }^{52}$, K.-A.Drees ${ }^{52}$, M.Dris $^{32}$, Y.Dufour ${ }^{9}$, F.Dupont ${ }^{14}$, D.Edsall ${ }^{1}$, R.Ehret ${ }^{17}$, G.Eigen ${ }^{4}$, T.Ekelof ${ }^{48}$, G.Ekspong ${ }^{44}$, M.Elsing ${ }^{52}$, J-P.Engel $^{10}$, N.Ershaidat ${ }^{23}$, B.Erzen ${ }^{43}$, M.Espirito Santo ${ }^{21}$, E.Falk ${ }^{24}$, D.Fassouliotis ${ }^{32}$, M.Feindt ${ }^{9}$, A.Ferrer ${ }^{49}$, T.A.Filippas ${ }^{32}$, A.Firestone ${ }^{1}$, P.-A.Fischer ${ }^{10}$, H.Foeth ${ }^{9}$, E.Fokitis ${ }^{32}$, F.Fontanelli ${ }^{13}$, F.Formenti $^{9}$, B.Franek ${ }^{37}$, P.Frenkiel $^{8}$, D.C.Fries ${ }^{17}$, A.G.Frodesen ${ }^{4}$, R.Fruhwirth ${ }^{50}$, F.Fulda-Quenzer ${ }^{19}$, J.Fuster ${ }^{49}$, A.Galloni ${ }^{22}$, D.Gamba $^{45}$, M.Gandelman $^{6}$, C.Garcia $^{49}$, J.Garcia ${ }^{41}$, C.Gaspar $^{9}$, U.Gasparini ${ }^{36}$, Ph.Gavillet ${ }^{9}$, E.N.Gazis ${ }^{32}$, D.Gele ${ }^{10}$, J-P.Gerber ${ }^{10}$, L.Gerdyukov ${ }^{42}$, M.Gibbs $^{22}$, R.Gokieli ${ }^{51}$, B.Golob ${ }^{43}$, G.Gopal ${ }^{37}$, L.Gorn ${ }^{1}$, M.Gorski ${ }^{51}$, Yu.Gouz ${ }^{45,42}$, V.Gracco ${ }^{13}$, E.Graziani ${ }^{40}$, G.Grosdidier ${ }^{19}$, K.Grzelak ${ }^{51}$, S.Gumenyuk ${ }^{28,53}$, P.Gunnarsson ${ }^{44}$, M.Gunther ${ }^{48}$, J.Guy ${ }^{37}$, F.Hahn ${ }^{9}$, S.Hahn ${ }^{52}$, Z.Hajduk ${ }^{18}$, A.Hallgren ${ }^{48}$, K.Hamacher ${ }^{52}$, W.Hao ${ }^{31}$, F.J.Harris ${ }^{35}$, V.Hedberg $^{24}$, R.Henriques ${ }^{21}$, J.J.Hernandez ${ }^{49}$, P.Herquet ${ }^{2}$, H.Herr ${ }^{9}$, T.L.Hessing ${ }^{9}$, E.Higon ${ }^{49}$, H.J.Hilke ${ }^{9}$, T.S.Hill $^{1}$, S-O.Holmgren ${ }^{44}$, P.J.Holt ${ }^{35}$, D.Holthuizen ${ }^{31}$, S.Hoorelbeke ${ }^{2}$, M.Houlden ${ }^{22}$, J.Hrubec ${ }^{50}$, K.Huet ${ }^{2}$, K.Hultqvist ${ }^{44}$, J.N.Jackson ${ }^{22}$, R.Jacobsson ${ }^{44}$, P.Jalocha ${ }^{18}$, R.Janik ${ }^{7}$, G.Jarlskog ${ }^{24}$. P.Jarry ${ }^{39}$, B.Jean-Marie ${ }^{19}$, E.K.Johansson ${ }^{44}$, L.Jonsson ${ }^{24}$, P.Jonsson ${ }^{24}$, C.Joram ${ }^{9}$, P.Juillot ${ }^{10}$, M.Kaiser $^{17}$, F.Kapusta ${ }^{23}$, K.Karafasoulis ${ }^{11}$, M.Karlsson ${ }^{44}$, E.Karvelas ${ }^{11}$, S.Katsanevas ${ }^{3}$, E.C.Katsoufis ${ }^{32}$, R.Keranen ${ }^{44}$, B.A.Khomenko ${ }^{16}$, N.N.Khovanski ${ }^{16}{ }$, B.King ${ }^{22}$, N.J.Kjaer ${ }^{9}$, H.Klein ${ }^{9}$, A.Klovning ${ }^{4}$, P.Kluit ${ }^{31}$, B.Koene ${ }^{31}$, P.Kokkinias ${ }^{11}$, M.Koratzinos ${ }^{9}$, V.Kostioukhine $^{42}$, C.Kourkoumelis ${ }^{3}$, O.Kouznetsov ${ }^{13}$, P.-H.Kramer ${ }^{52}$, M.Krammer ${ }^{50}$, C.Kreuter $^{17}$, J.Krolikowski $^{51}$, I.Kronkvist ${ }^{24}$, Z.Krumstein ${ }^{16}$, W.Krupinski ${ }^{18}$, P.Kubinec ${ }^{7}$, W.Kucewicz ${ }^{18}$, K.Kurvinen ${ }^{15}$, C.Lacasta $^{49}$, I.Laktineh ${ }^{25}$, S.Lamblot ${ }^{23}$, J.W.Lamsa ${ }^{1}$, L.Lanceri ${ }^{46}$, D.W.Lane ${ }^{1}$, P.Langefeld ${ }^{52}$, V.Lapin $^{42}{ }^{4}$, I.Last $^{22}$, J-P.Laugier ${ }^{39}$, R.Lauhakangas ${ }^{15}$, G.Leder ${ }^{50}$, F.Ledroit ${ }^{14}$, V.Lefebure ${ }^{2}$, C.K.Legan ${ }^{1}$, R.Leitner ${ }^{30}$, Y.Lemoigne $^{39}$, J.Lemonne ${ }^{2}$, G.Lenzen ${ }^{52}$, V.Lepeltier ${ }^{19}$, T.Lesiak ${ }^{36}$, D.Liko ${ }^{50}$, R.Lindner ${ }^{52}$, A.Lipniacka ${ }^{36}$, I.Lippi $^{36}$, B.Loerstad ${ }^{24}$, M.Lokajicek ${ }^{12}$, J.G.Loken ${ }^{35}$, J.M.Lopez ${ }^{41}$, A.Lopez-Fernandez ${ }^{9}$, M.A.Lopez Aguera ${ }^{41}$, D.Loukas ${ }^{11}$, P.Lutz ${ }^{39}$, L.Lyons ${ }^{35}$, J.MacNaughton ${ }^{50}$, G.Maehlum ${ }^{17}$, A.Maio ${ }^{21}$, V.Malychev ${ }^{16}$, F.Mand ${ }^{50}$, C.Maocun $^{2}$, J.Marco ${ }^{41}$, B.Marechal ${ }^{47}$, M.Margoni ${ }^{36}$, J-C.Marin ${ }^{9}$, C.Mariotti ${ }^{40}$, A.Markou ${ }^{11}$, T.Maron $^{52}$, C.Martinez-Rivero $^{41}$, F.Martinez-Vidal ${ }^{49}$, S.Marti i Garcia ${ }^{49}$, F.Matorras ${ }^{41}$, C.Matteuzzi ${ }^{28}$, G.Matthiae ${ }^{38}$, M.Mazzucato $^{36}$, M.Mc Cubbin ${ }^{9}$, R.Mc Kay ${ }^{1}$, R.Mc Nulty ${ }^{22}$, J.Medbo ${ }^{48}$, C.Meroni ${ }^{28}$, S.Meyer ${ }^{17}$, W.T.Meyer ${ }^{1}$, M.Michelotto $^{36}$, E.Migliore ${ }^{45}$, L.Mirabito ${ }^{25}$, W.A.Mitaroff ${ }^{50}$, U.Mjoernmark ${ }^{24}$, T.Moa ${ }^{44}$, R.Moeller ${ }^{29}$, K.Moenig $^{9}$, M.R.Monge ${ }^{13}$, P.Morettini ${ }^{13}$, H.Mueller ${ }^{17}$, L.M.Mundim ${ }^{6}$, W.J.Murray ${ }^{37}$, B.Muryn ${ }^{18}$, G.Myatt $^{35}$, F.Naraghi ${ }^{14}$, F.L.Navarria ${ }^{5}$, S.Navas ${ }^{49}$, P.Negri ${ }^{28}$, S.Nemecek ${ }^{12}$, W.Neumann ${ }^{52}$, N.Neumeister ${ }^{50}$, R.Nicolaidou ${ }^{3}$, B.S.Nielsen ${ }^{29}$, M.Nieuwenhuizen ${ }^{31}$, V.Nikolaenko ${ }^{10}$, P.Niss ${ }^{44}$, A.Nomerotski ${ }^{36}$, A.Normand ${ }^{35}$, W.Oberschulte-Beckmann ${ }^{17}$, V.Obraztsov ${ }^{42}$, A.G.Olshevski ${ }^{16}$, A.Onofre ${ }^{21}$, R.Orava ${ }^{15}$, K.Osterberg ${ }^{15}$, A.Ouraou $^{39}$, P.Paganini ${ }^{19}$, M.Paganoni ${ }^{9}$, P.Pages ${ }^{10}$, H.Palka ${ }^{18}$, Th.D.Papadopoulou ${ }^{32}$, K.Papageorgiou ${ }^{11}$, L.Pape $^{9}$, C.Parkes ${ }^{35}$, F.Parodi ${ }^{13}$, A.Passeri ${ }^{40}$, M.Pegoraro ${ }^{36}$, L.Peralta ${ }^{21}$, M.Pernicka ${ }^{50}$, A.Perrotta ${ }^{5}$, C.Petridou $^{46}$, A.Petrolini ${ }^{13}$, M.Petrovyck ${ }^{28,53}$, H.T.Phillips ${ }^{37}$, G.Piana ${ }^{13}$, F.Pierre ${ }^{39}$, M.Pimenta ${ }^{21}$, M.Pindo ${ }^{28}$, S.Plaszczynski ${ }^{19}$, O.Podobrin ${ }^{17}$, M.E.Pol ${ }^{6}$, G.Polok ${ }^{18}$, P.Poropat ${ }^{46}$, V.Pozdniakov ${ }^{16}$, M.Prest ${ }^{46}$, P.Privitera ${ }^{38}$, N.Pukhaeva ${ }^{16}$, D.Radojicic ${ }^{35}$, S.Ragazzi ${ }^{28}$, H.Rahmani $^{32}$, J.Rames ${ }^{12}$, P.N.Ratoff ${ }^{20}$, A.L.Read ${ }^{33}$, M.Reale ${ }^{52}$, P.Rebecchi $^{19}$, N.G.Redaelli ${ }^{28}$, M.Regler ${ }^{50}$, D.Reid ${ }^{9}$, P.B.Renton ${ }^{35}$, L.K.Resvanis ${ }^{3}$, F.Richard ${ }^{19}$, J.Richardson $^{22}$, J.Ridky $^{12}$, G.Rinaudo ${ }^{45}$, I.Ripp ${ }^{39}$, A.Romero ${ }^{45}$, I.Roncagliolo ${ }^{13}$, P.Ronchese ${ }^{36}$, L.Roos ${ }^{14}$, E.I.Rosenberg ${ }^{1}$, E.Rosso $^{9}$, P.Roudeau ${ }^{19}$, T.Rovelli ${ }^{5}$, W.Ruckstuhl ${ }^{31}$, V.Ruhlmann-Kleider ${ }^{39}$, A.Ruiz ${ }^{41}$, K.Rybicki ${ }^{18}$, H.Saarikko ${ }^{15}$, Y.Sacquin ${ }^{39}$, A.Sadovsky ${ }^{16}$, G.Sajot $^{14}$, J.Salt ${ }^{49}$, J.Sanchez ${ }^{26}$, M.Sannino ${ }^{13}$, M.Schimmelpfennig ${ }^{17}$, H.Schneider ${ }^{17}$, U.Schwickerath ${ }^{17}$, M.A.E.Schyns ${ }^{52}$, G.Sciolla ${ }^{45}$, F.Scuri ${ }^{46}$, P.Seager ${ }^{20}$, Y.Sedykh ${ }^{16}$, A.M.Segar ${ }^{35}$,

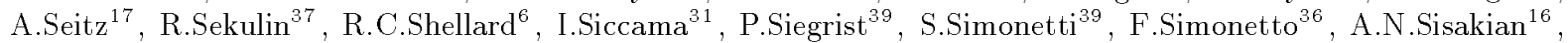
B.Sitar ${ }^{7}$, T.B.Skaali ${ }^{33}$, G.Smadja ${ }^{25}$, N.Smirnov ${ }^{42}$, O.Smirnova ${ }^{16}$, G.R.Smith ${ }^{37}$, R.Sosnowski ${ }^{51}$, D.Souza-Santos ${ }^{6}$, T.Spassov ${ }^{21}$, E.Spiriti ${ }^{40}$, P.Sponholz ${ }^{52}$, S.Squarcia ${ }^{13}$, C.Stanescu ${ }^{40}$, S.Stapnes ${ }^{33}$, I.Stavitski ${ }^{36}$, F.Stichelbaut $^{9}$, A.Stocchi ${ }^{19}$, J.Strauss ${ }^{50}$, R.Strub ${ }^{10}$, B.Stugu ${ }^{4}$, M.Szczekowski ${ }^{51}$, M.Szeptycka ${ }^{51}$, T.Tabarelli ${ }^{28}{ }^{\text {, }}$ 
J.P.Tavernet $^{23}$, O.Tchikilev ${ }^{42}$, A.Tilquin ${ }^{27}$, J.Timmermans ${ }^{31}$, L.G.Tkatchev ${ }^{16}$, T.Todorov ${ }^{10}$, D.Z.Toet ${ }^{31}$, A.Tomaradze $^{2}$, B.Tome ${ }^{21}$, L.Tortora ${ }^{40}$, G.Transtromer ${ }^{24}$, D.Treille ${ }^{9}$, W.Trischuk ${ }^{9}$, G.Tristram ${ }^{8}$, A.Trombini ${ }^{19}{ }^{9}$, C.Troncon $^{28}$, A.Tsirou ${ }^{9}$, M-L.Turluer ${ }^{39}$, I.A.Tyapkin ${ }^{16}$, M.Tyndel ${ }^{37}$, S.Tzamarias ${ }^{22}$, B.Ueberschaer ${ }^{52}$, O.Ullaland ${ }^{9}$, V.Uvarov ${ }^{42}$, G.Valenti ${ }^{5}$, E.Vallazza ${ }^{9}$, C.Vander Velde ${ }^{2}$, G.W.Van Apeldoorn ${ }^{31}$, P.Van Dam ${ }^{31}$, W.K.Van Doninck ${ }^{2}$, J.Van Eldik ${ }^{31}$, N.Vassilopoulos ${ }^{35}$, G.Vegni ${ }^{28}$, L.Ventura ${ }^{36}$, W.Venus ${ }^{37}$, F.Verbeure ${ }^{2}$, M.Verlato $^{36}$, L.S.Vertogradov ${ }^{16}$, D.Vilanova ${ }^{39}$, P.Vincent ${ }^{25}$, L.Vitale ${ }^{46}$, E.Vlasov ${ }^{42}$, A.S.Vodopyanov $^{16}$, V.Vrba $^{12}$, H.Wahlen ${ }^{52}$, C.Walck ${ }^{44}$, M.Weierstall ${ }^{52}$, P.Weilhammer ${ }^{9}$, C.Weiser ${ }^{17}$, A.M.Wetherell ${ }^{9}$, D.Wicke ${ }^{52}$, J.H.Wickens ${ }^{2}$, M.Wielers ${ }^{17}$, G.R.Wilkinson ${ }^{35}$, W.S.C.Williams ${ }^{35}$, M.Winter ${ }^{10}$, M.Witek $^{18}$, K.Woschnagg $^{48}$, K.Yip ${ }^{35}$, O.Yushchenko ${ }^{42}$, F.Zach ${ }^{25}$, C.Zacharatou ${ }^{24}$, A.Zaitsev ${ }^{42}$, A.Zalewska ${ }^{18}$, P.Zalewski ${ }^{51}$, D.Zavrtanik ${ }^{43}$, E.Zevgolatakos ${ }^{11}$, N.I.Zimin ${ }^{16}$, M.Zito ${ }^{39}$, D.Zontar ${ }^{43}$, R.Zuberi ${ }^{35}$, G.C.Zucchelli ${ }^{44}$, G.Zumerle ${ }^{36}$

\footnotetext{
${ }^{1}$ Ames Laboratory and Department of Physics, Iowa State University, Ames IA 50011, USA

${ }^{2}$ Physics Department, Univ. Instelling Antwerpen, Universiteitsplein 1, B-2610 Wilrijk, Belgium and IIHE, ULB-VUB, Pleinlaan 2, B-1050 Brussels, Belgium

and Faculté des Sciences, Univ. de l'Etat Mons, Av. Maistriau 19, B-7000 Mons, Belgium

${ }^{3}$ Physics Laboratory, University of Athens, Solonos Str. 104, GR-10680 Athens, Greece

${ }^{4}$ Department of Physics, University of Bergen, Allégaten 55, N-5007 Bergen, Norway

${ }^{5}$ Dipartimento di Fisica, Università di Bologna and INFN, Via Irnerio 46, I-40126 Bologna, Italy

${ }^{6}$ Centro Brasileiro de Pesquisas Físicas, rua Xavier Sigaud 150, RJ-22290 Rio de Janeiro, Brazil and Depto. de Física, Pont. Univ. Católica, C.P. 38071 RJ-22453 Rio de Janeiro, Brazil and Inst. de Física, Univ. Estadual do Rio de Janeiro, rua São Francisco Xavier 524, Rio de Janeiro, Brazil ${ }^{7}$ Comenius University, Faculty of Mathematics and Physics, Mlynska Dolina, SK-84215 Bratislava, Slovakia

${ }^{8}$ Collège de France, Lab. de Physique Corpusculaire, IN2P3-CNRS, F-75231 Paris Cedex 05, France

${ }^{9} \mathrm{CERN}, \mathrm{CH}-1211$ Geneva 23, Switzerland

${ }^{10}$ Centre de Recherche Nucléaire, IN2P3 - CNRS/ULP - BP20, F-67037 Strasbourg Cedex, France

${ }^{11}$ Institute of Nuclear Physics, N.C.S.R. Demokritos, P.O. Box 60228, GR-15310 Athens, Greece

${ }^{12}$ FZU, Inst. of Physics of the C.A.S. High Energy Physics Division, Na Slovance 2, 180 40, Praha 8, Czech Republic

${ }^{13}$ Dipartimento di Fisica, Università di Genova and INFN, Via Dodecaneso 33, I-16146 Genova, Italy

${ }^{14}$ Institut des Sciences Nucléaires, IN2P3-CNRS, Université de Grenoble 1, F-38026 Grenoble Cedex, France

${ }^{15}$ Research Institute for High Energy Physics, SEFT, P.O. Box 9, FIN-00014 Helsinki, Finland

${ }^{16}$ Joint Institute for Nuclear Research, Dubna, Head Post Office, P.O. Box 79, 101000 Moscow, Russian Federation

${ }^{17}$ Institut für Experimentelle Kernphysik, Universität Karlsruhe, Postfach 6980, D-76128 Karlsruhe, Germany

${ }^{18}$ Institute of Nuclear Physics and University of Mining and Metalurgy, Ul. Kawiory 26a, PL-30055 Krakow, Poland

${ }^{19}$ Université de Paris-Sud, Lab. de l'Accélérateur Linéaire, IN2P3-CNRS, Bât. 200, F-91405 Orsay Cedex, France

${ }^{20}$ School of Physics and Materials, University of Lancaster, Lancaster LA1 4YB, UK

${ }^{21}$ LIP, IST, FCUL - Av. Elias Garcia, $14-1^{\circ}$, P-1000 Lisboa Codex, Portugal

${ }^{22}$ Department of Physics, University of Liverpool, P.O. Box 147, Liverpool L69 3BX, UK

${ }^{23}$ LPNHE, IN2P3-CNRS, Universités Paris VI et VII, Tour 33 (RdC), 4 place Jussieu, F-75252 Paris Cedex 05, France

${ }^{24}$ Department of Physics, University of Lund, Sölvegatan 14, S-22363 Lund, Sweden

${ }^{2.5}$ Université Claude Bernard de Lyon, IPNL, IN2P3-CNRS, F-69622 Villeurbanne Cedex, France

${ }^{26}$ Universidad Complutense, Avda. Complutense s/n, E-28040 Madrid, Spain

${ }^{27}$ Univ. d'Aix - Marseille II - CPP, IN2P3-CNRS, F-13288 Marseille Cedex 09, France

${ }^{28}$ Dipartimento di Fisica, Università di Milano and INFN, Via Celoria 16, I-20133 Milan, Italy

${ }^{29}$ Niels Bohr Institute, Blegdamsvej 17, DK-2100 Copenhagen 0, Denmark

${ }^{30} \mathrm{NC}$, Nuclear Centre of MFF, Charles University, Areal MFF, V Holesovickach 2, 180 00, Praha 8, Czech Republic

${ }^{31}$ NIKHEF-H, Postbus 41882, NL-1009 DB Amsterdam, The Netherlands

${ }^{32}$ National Technical University, Physics Department, Zografou Campus, GR-15773 Athens, Greece

${ }^{33}$ Physics Department, University of Oslo, Blindern, N-1000 Oslo 3, Norway

${ }^{34}$ Dpto. Fisica, Univ. Oviedo, C/P. Pérez Casas, S/N-33006 Oviedo, Spain

${ }^{35}$ Department of Physics, University of Oxford, Keble Road, Oxford OX1 3RH, UK

${ }^{36}$ Dipartimento di Fisica, Università di Padova and INFN, Via Marzolo 8, I-35131 Padua, Italy

${ }^{37}$ Rutherford Appleton Laboratory, Chilton, Didcot OX11 OQX, UK

${ }^{38}$ Dipartimento di Fisica, Università di Roma II and INFN, Tor Vergata, I-00173 Rome, Italy

${ }^{39}$ Centre d'Etudes de Saclay, DSM/DAPNIA, F-91191 Gif-sur-Yvette Cedex, France

${ }^{40}$ Istituto Superiore di Sanità, Ist. Naz. di Fisica Nucl. (INFN), Viale Regina Elena 299, I-00161 Rome, Italy

${ }^{41}$ Instituto de Fisica de Cantabria (CSIC-UC), Avda. los Castros, S/N-39006 Santander, Spain, (CICYT-AEN93-0832)

${ }^{42}$ Inst. for High Energy Physics, Serpukov P.O. Box 35, Protvino, (Moscow Region), Russian Federation

${ }^{43} \mathrm{~J}$. Stefan Institute and Department of Physics, University of Ljubljana, Jamova 39, SI-61000 Ljubljana, Slovenia

${ }^{44}$ Fysikum, Stockholm University, Box 6730, S-113 85 Stockholm, Sweden

${ }^{45}$ Dipartimento di Fisica Sperimentale, Università di Torino and INFN, Via P. Giuria 1, I-10125 Turin, Italy

${ }^{46}$ Dipartimento di Fisica, Università di Trieste and INFN, Via A. Valerio 2, I-34127 Trieste, Italy and Istituto di Fisica, Università di Udine, I-33100 Udine, Italy

${ }^{47}$ Univ. Federal do Rio de Janeiro, C.P. 68528 Cidade Univ., Ilha do Fundão BR-21945-970 Rio de Janeiro, Brazil

${ }^{48}$ Department of Radiation Sciences, University of Uppsala, P.O. Box 535, S-751 21 Uppsala, Sweden

${ }^{49}$ IFIC, Valencia-CSIC, and D.F.A.M.N., U. de Valencia, Avda. Dr. Moliner 50, E-46100 Burjassot (Valencia), Spain

${ }^{50}$ Institut für Hochenergiephysik, Österr. Akad. d. Wissensch., Nikolsdorfergasse 18, A-1050 Vienna, Austria

${ }^{51}$ Inst. Nuclear Studies and University of Warsaw, Ul. Hoza 69, PL-00681 Warsaw, Poland

${ }^{52}$ Fachbereich Physik, University of Wuppertal, Postfach 100 127, D-42097 Wuppertal 1, Germany

${ }^{53}$ On leave of absence from IHEP Serpukhov
} 


\section{Introduction}

This paper presents a measurement of the inclusive $\pi^{0}$ cross section in hadronic $Z^{0} \rightarrow$ $q \bar{q}$ and $Z^{0} \rightarrow b \bar{b}$ decays with the DELPHI detector. Information about the fragmentation of quarks and gluons into hadrons can be extracted from the composition of hadronic particles in the $q \bar{q}$ final state in $e^{+} e^{-}$annihilation. The first phase of this annihilation process can be calculated by perturbation theory. The subsequent fragmentation into hadrons is described only by phenomenological models which are implemented in event generators.

In this paper, the measured inclusive $\pi^{0}$ cross sections in hadronic events and those tagged as having a B decay have been compared with the predictions of different Monte Carlo event generators with optimized parameters [1]. The tuning is based only on charged tracks and the branching ratios are taken from [2]. The following generators have been used: JETSET 7.4 [3] where the LUND string model is implemented; ARIADNE 4.6 [4] which uses the colour dipole model for QCD cascades followed by the same 'string' fragmentation as implemented in JETSET and HERWIG 5.8C [5] which is based on the formation and decay of colourless clusters. JETSET, ARIADNE and HERWIG throughout this paper stand for the event generators with parameters adjusted according to previous QCD studies [1] unless otherwise indicated. Furthermore, the measured spectrum is displayed as function of $\xi_{p}=\ln \left(1 / x_{p}\right)$ and $x_{p}=2 \cdot p / \sqrt{s}$. In this variable the spectrum has a broad Gaussian shape at large values of $\xi_{p}$ (i.e. small momenta). The peak position is a function of the centre of mass energy and the particle mass as predicted e.g. by the modified leading $\log$ approximation (MLLA) [6]. The average number of neutral pions coming from B-hadrons is determined.

\section{Apparatus and Data Selection}

A detailed description of the DELPHI detector can be found in reference [7]. The main parts of the detector used for this analysis are the barrel electromagnetic calorimeter (HPC), for the shower reconstruction and the Time Projection Chamber (TPC), for the reconstruction of electron tracks from photon conversions. In addition the vertex detector (VD) is important for tagging events with $\mathrm{B}$ decays. The $\mathrm{z}$ axis is defined as the electron beam direction, and the $\mathrm{R} \phi$ plane is transverse to it at the collision point. $\theta$ and $\phi$ are the usual polar and azimuthal angles in this frame.

The HPC [8,9] consists of 144 modules arranged in 6 rings inside the magnetic field covering the polar angular region between $43^{\circ}$ and $137^{\circ}$. Each ring consists of 24 modules arranged concentrically around the beam axis with an inner radius of $208 \mathrm{~cm}$ and an outer radius of $260 \mathrm{~cm}$. Each $\mathrm{HPC}$ module is a time projection chamber with 40 layers of lead $(\mathrm{Pb})$ in the gas volume which serve not only as converter material, but also form the drift field. The converter thickness is 18 radiation lengths. In each module there are 128 pads arranged in 9 (staggered) pad rows, each being read out using 256 FADC samples per event at $15 \mathrm{MHz}$ which provides a resolution of $3.5 \mathrm{~mm}$ along the beam axis. The TPC is the main tracking chamber in DELPHI which is working on the time projection principle. It covers the polar angular region between $20^{\circ}$ and $160^{\circ}$ and is situated at $29 \mathrm{~cm}<R<122 \mathrm{~cm}$. The spatial resolution for tracks from hadronic $Z^{0}$ decays is $250 \mu \mathrm{m}$ in the $\mathrm{R} \phi$ plane perpendicular to the beam direction and $880 \mu \mathrm{m}$ in the $\mathrm{Rz}$ plane parallel to the beam direction. The DELPHI vertex detector is a silicon detector giving a high precision measurement of the position of charged particles near the interaction point. It consists of three concentric shells of Si-strip detectors at radii of 
$6.3,9$ and $11 \mathrm{~cm}$. The readout pitch is $50 \mu \mathrm{m}$ in the $\mathrm{R} \phi$ plane. Since 1994, the first and third layers have been equipped with double sided silicon detectors, giving measurements also in the Rz plane.

After standard barrel hadronic selections on the DELPHI data [10] from the 1992 to 1994 running periods and excluding runs with known TPC or HPC problems, in which less than $95 \%$ of the detector is operating correctly, there are 477621 events from 1992 , 501212 events from 1993 and 975435 events from 1994, for a total of 1954268 events. About twice as many simulation events were used in this analysis. Due to changes in the HPC pattern recognition algorithm the 1994 data are treated separately from the 1992 and 1993 data. Throughout this paper the final results are obtained by taking the weighted mean of results from 1992-1993 and 1994 samples.

To calculate the efficiencies and purities for the current analysis, the JETSET 7.3 Monte Carlo generator [3] is used and the detector response is simulated with the DELSIM package[11]. Finally, the Monte Carlo data are processed through the same reconstruction and analysis programs as the real data.

In order to enrich the sample of $b \bar{b}$ events, a technique that capitalizes on the distinct differences in mass and lifetime between $\mathrm{B}$ hadrons and those hadrons made with lighter quarks is used [12]. B mesons are nearly three times heavier than D mesons and have lifetimes that are more than 50\% longer. Also, the energy spectrum of the $\mathrm{B}$ meson is generally harder than that of primary D mesons, and the B mainly decays into a D meson. These features result in a distribution of impact parameters that is typically larger in the $B$ meson events than in events without $B$ mesons. For each event the probability that all tracks with positive impact parameters originate from a single vertex compatible with the beam spot is calculated. The impact parameter is positive if the vector joining the primary vertex and the point of closest approach to the track lies in the same direction as the jet to which the given track belongs. Selecting only those events where this probability is less than $1 \%$ results in an efficiency of $(54.6 \pm 0.4) \%$ and a purity of $(81.8 \pm 0.4) \%$ for $b \bar{b}$ events in the 1992 data. For 1993 the efficiency is $(60.0 \pm 0.4) \%$ and the purity is $(82.2 \pm 0.4) \%$. For 1994 the efficiency is $(62.1 \pm 0.4) \%$ and the purity $(80.9 \pm 0.4) \%$. This b quark enhanced sample consists of 73268 events taken in 1992, 99262 events in 1993 and 162900 events in 1994.

\section{Photon reconstruction}

Two different photon reconstruction methods are used in this analysis: reconstruction of electromagnetic showers initiated by neutral particles in the HPC, and the reconstruction of photons which convert in the material in front of the sensitive volume of the DELPHI TPC. In the barrel region $7 \%$ of all photons convert in front of the TPC. These conversions can be reconstructed with a very good precision on the energy. Before reaching the HPC approximately $42 \% / \sin \theta$ of all photons convert so that only $59 \%$ of all real photons reach the HPC and look like one shower.

\subsection{Converted photons}

Photon conversions in front of the TPC are reconstructed by an algorithm that examines charged tracks reconstructed in the TPC. Unlike most pair finding algorithms, it first analyses single tracks. Each TPC track is examined for a point $\mathrm{P}$, where the tangent to the helix (in the $R \phi$ projection i.e. in the plane perpendicular to the beam) is pointing directly to the main vertex defined by the beam spot position. Under the assumption 
that the opening angle of the electron-positron pair is zero, one finds with this procedure the conversion radius $R$, i.e. the transverse distance from the main vertex to the point $P$. For particles originating from the main vertex, the condition is satisfied for $\mathrm{R}=0$, i.e. within the experimental accuracy it is either a small $\mathrm{R}$ or no solution exists. In the following, all tracks which have a solution $\mathrm{R}$ with $R / \sigma(R)>1$ are accepted as conversion candidates. Since the curvature decreases with increasing energy, highly energetic tracks are often compatible with both the main vertex and a secondary vertex. The one standard deviation cut is necessary to keep background at a tolerable level, but it limits the efficiency at high energies.

If two oppositely-charged conversion candidates are found with compatible decay point parameters, they are accepted as a converted photon. The following selection criteria are imposed:

1. The weighted mean conversion radius of both tracks is at least one standard deviation away from the main vertex (defined by the beam spot).

2. The reconstructed mean conversion radius is at least $5 \mathrm{~cm}$ and below $50 \mathrm{~cm}$ (before the main TPC gas volume).

3. At least one of the tracks has no associated point before the reconstructed mean conversion radius.

4. The difference in azimuthal angle $\Delta \phi$ of both conversion points is below $30 \mathrm{mrad}$.

5. The difference in polar angle $\Delta \theta$ of the two tracks is below $15 \mathrm{mrad}$.

If these criteria are fulfilled, a $\chi^{2}$ is calculated from $\Delta \phi, \Delta \theta$ and the difference of the reconstructed conversion radii $\Delta R$ in order to find the best combinations in cases where there are ambiguous associations. The energy of the conversion electrons is corrected for radiation losses by a factor that depends on the amount of material between the conversion point and the entrance to the TPC. The reconstructed corrected photons with an acceptable $\chi^{2}$ have a precision on their energy of $\pm 1.2 \%$, an angular resolution of \pm 1.5 $\operatorname{mrad}$ in $\theta$ and $\phi$, and a precision on the conversion radius of $\pm 5 \mathrm{~mm}$. These values have been obtained from simulation.

The algorithm outlined above becomes inefficient for high or low momentum particles. For high momentum particles, the conversion points are poorly determined, while particles with transverse momentum relative to the beam axis less than $50 \mathrm{MeV} / \mathrm{c}$ do not reach the TPC. Therefore, two additional algorithms are applied. For high momentum particles, a pair-finding algorithm which fits two oppositely charged conversion pairs to the same vertex is used. A precision on the energy of $\pm 1.8 \%$ is achieved with an angular precision of $\pm 1.5 \mathrm{mrad}$ in $\theta$ and $\phi$. These values have been obtained from simulation.

Some conversions leave only single tracks in the TPC, while one of the tracks may be lost inside the beampipe or not be reconstructed because of too few hits in the TPC. These single tracks are accepted as conversions only if the following conditions are fulfilled:

1. The conversion radius is between 22 and $33 \mathrm{~cm}$.

2. In the $R \phi$ plane the distance of the conversion radius from the main vertex is at least 4 standard deviations $(R / \sigma(R)>4)$.

3 . No hits are associated in front of the reconstructed conversion point.

4. The z-coordinate of the conversion point and that from the angular extrapolation from the reconstructed primary vertex towards the conversion point coincide to within $1 \mathrm{~cm}$.

5. The reconstructed photon energy divided by $\sin \theta$ is below $5 \mathrm{GeV}\left(E_{\gamma} / \sin \theta<\right.$ $5 \mathrm{GeV})$. 
From the simulation a precision on the photon energy of $\pm 12 \%$ is obtained after applying a mean energy correction for the unseen lepton and an angular precision of $\pm 1.5 \mathrm{mrad}$ in $\theta$ and $\phi$.

Reasonable agreement between data and simulation is observed in the distribution of the reconstructed conversion radii, as shown in figure 1. However, some discrepancies are found around $R=30 \mathrm{~cm}$ and are discussed later. The energy spectra of converted photons for simulated and real data are shown in figure 2. Note that the number of reconstructed photons converted before the TPC is an order of magnitude lower than the number of photons reconstructed in the HPC. Nevertheless, the statistics are large enough to provide a good estimate of the systematic errors by comparing the $\pi^{0}$ reconstruction efficiencies from the various photon samples.

\subsection{Calorimetric photons}

Showers in the HPC are reconstructed by summing neighbouring clusters (measured in 3 dimensions) with a lateral spread of up to $\pm 2^{0}$ in $\phi$ and $\pm 1^{0}$ in $\theta$ [9]. A cluster is defined as the sum of the charge obtained during a given drift time interval. If the width along the beam axis of a cluster is large a higher threshold is applied and the cluster is split into smaller clusters. The precision on the measured energy is $31 \% / E^{0.44} \oplus 2.7 \%$ which was deduced from the width of the $\pi^{0}$ peaks reconstructed by one converted and one HPC photon. For HPC photons with less than $2 \mathrm{GeV}$ energy the the $\pi^{0}$ peak width is dominated by the precision on the energy of the HPC photon and the contribution of the precision of the energy of the converted photon can be neglected. For Bhabha energies the same precision on the energy for charged and neutral showers is assumed. From the simulation the angular precision is measured to be $\pm 1.0 \mathrm{mrad}$ in polar angle $(\theta)$ and $\pm 1.7 \mathrm{mrad}$ in azimuthal angle $(\phi)$.

A neutral shower is a shower which cannot be associated with a TPC track. Due to the amount of material in front of the HPC (beam pipe, Vertex Detector, Inner Detector, TPC inner and outer wall, RICH inner wall, drift tube, mirrors and outer wall and Outer Detector) $42 \% / \sin \theta$ of the photons convert before reaching the HPC. The presence of RICH end-flanges increases this number to around $80 \%$ in front of the outermost HPC rings. Therefore this analysis of HPC showers is limited to the four innermost rings, i.e. the region with $|\cos \theta|<0.65$. Photons converting after the TPC reach the calorimeter as several showers separated in $\phi$ by the magnetic field.

A fraction of these conversions can be recognized by track elements in the outer detector just in front of the HPC, thus reducing the combinatorial background for the $\pi^{0}$ reconstruction. There are some small differences in the HPC shower energies between data and simulation, which can be attributed to the presence of low energy electrons curling in the HPC drift channels, which are less abundant in the simulation due to the relatively high energy cut-offs of the shower evolution inside the HPC by the EGS program [13]. These effects have been accounted for. Only HPC showers passing the longitudinal and transverse shower profile cuts are used in the analysis. Photons recognized as electron radiation and showers near the module edges are excluded. A good agreement is found between the obtained photon spectra for real and simulated data which can be seen in figure 2. 


\section{$4 \quad \pi^{0}$ Reconstruction}

Having reconstructed 'HPC' and 'converted' photons, $\pi^{0}$ 's can be reconstructed by calculating the invariant $\gamma \gamma$ mass. Combining HPC and converted photons gives three different $\pi^{0}$ reconstruction methods: pairs of converted photons; pairs of one converted and one HPC photon which are used for low and intermediate $\pi^{0}$ energies $(0.5-15 \mathrm{GeV})$ and pairs of HPC photons which are used in the $\pi^{0}$ energy range from 3 to $8 \mathrm{GeV}$. At high energies $\left(6 \mathrm{GeV}<E_{\pi^{0}}<34 \mathrm{GeV}\right)$ the opening angle between the decay photons of $\pi^{0}$ s is so small that they are seen as one single shower in the calorimeter. In this energy range $\pi^{0}$ 's can be reconstructed by looking for substructures within one shower. Most of the high energy showers made by neutral particles in the HPC are in reality $\pi^{0}$ 's, as can be deduced from the simulation. Hence as a crosscheck the $\pi^{0}$ cross sections can be determined from all-neutral showers without looking for substructures.

\subsection{Low and intermediate energy $\pi^{0}$ reconstruction}

Figure 3a shows the invariant mass spectrum of the combination of two converted photons, figure $3 \mathrm{~b}$ from the combination of a converted photon with a HPC photon and figure $3 \mathrm{c}$ from the combination of two HPC photons in the energy range 4 to $8 \mathrm{GeV}$. There are clear $\pi^{0}$ signals on top of a combinatorial background which is increasing with the distance of the photon reconstruction from the interaction point (and decreasing energy). The background for very low invariant mass values in figure 3a is mainly arising from particles wrongly reconstructed as single conversions. The corresponding plots from the simulation look similar, with the background level and shape well described. As a cross check for these reconstruction methods, the angular decay distribution for the photons in the $\pi^{0}$ rest frame is examined. For a scalar particle, this distribution is predicted to be isotropic. For the combination of two converted photons and one converted and one calorimetric photon this distribution is shown in figure 4 . In both cases a flat distribution is found.

The $\pi^{0}$ peak position and width for the sample containing one conversion and one HPC photon offers the opportunity to understand the systematics of the HPC at low energies, since the converted photons are well measured in this case. The detection efficiency, as estimated from the simulation for the 1992 and 1993 data, is shown in figure 5 as function of $x_{p}$. The efficiency is calculated by the number of reconstructed $\pi^{0} \mathrm{~s}$ in a $x_{p}$ bin after the photon selection cuts mentioned above divided by the total number of generated $\pi^{0}$ 's in the same geometrical acceptance $(|\cos \theta|<0.65)$. The reconstruction efficiency for $\pi^{0}$ 's reconstructed by two converted photons or one converted photon and one calorimetric photon (see figures $5 \mathrm{a}$ and $5 \mathrm{~b}$ ) is falling towards very low $x_{p}$, because the tracks from the converted photons have insufficient energy to be reconstructed in the TPC. For higher $x_{p}$ the efficiency decreases because the curvature of the electron and positron tracks from conversions is too small and therefore these particles are not accepted as conversion candidates (see section 3.1 ). The efficiency for the $\pi^{0}$ reconstruction from two converted photons or from one converted and one HPC photon is about a factor 1.25 or 1.10 respectively larger in the 1994 data than in 1992-1993 due to the changes in the HPC pattern recognition algorithms. These numbers include the fraction of photons converting and the efficiency for reconstructing conversions and HPC showers and identifying them as a photon. 
Although some efficiencies are small, they are determined reliably using simulated events since the largest losses are due to geometrical acceptance and material distribution. Several cross checks on e.g. the conversion radius distribution, the ratio between single and double track conversions have been performed, which show an agreement between data and simulation. The corresponding uncertainties are discussed in section 5 .

\subsection{High energy $\pi^{0}$ reconstruction}

The opening angle between the decay photons of $\pi^{0}$ 's with energies larger than $6 \mathrm{GeV}$ is generally less than $2^{0}$. In this case, the HPC pattern recognition program reconstructs single or overlapping showers. For the identification of $\pi^{0}$ s the very fine granularity of the HPC permits a search for a lateral substructure in HPC showers with reconstructed energies larger than $6 \mathrm{GeV}$. The sampling width in the drift direction $(\mathrm{z})$ corresponds to $3.5 \mathrm{~mm}$, whereas the typical pad width in the first layers is $2-3 \mathrm{~cm}$. In the algorithm to find substructures all cluster measurements are projected on a $\theta-\phi$ grid where $\theta$ and $\phi$ are the usual polar angles. A $\theta$ bin is chosen to correspond to a time slot. The charge of each $\theta$ bin of a pad is equally distributed into $\phi$ bins of the same size as the $\theta$ bins. The charges of all pads belonging to the shower are distributed over this grid and added using a weight depending on the depth of the pad row. The weights were optimized to achieve the best two-shower separation, i.e. the pad-rows containing the start of the shower evolution get the highest weights. Examples of the resulting charge distribution for a single photon of $25 \mathrm{GeV}$ (from a $Z^{0} \rightarrow \mu^{+} \mu^{-} \gamma$ event) and a $\pi^{0}$ of $29 \mathrm{GeV}$ (from a $\tau^{-} \rightarrow \nu_{\tau} \rho^{-} \rightarrow \nu_{\tau} \pi^{-} \pi^{0}$ decay) are shown in figure 6 .

The next step consists in finding the eigenvalues and eigenvectors of the lateral charge distribution tensor based on $\theta_{i}, \phi_{i}$ and the corresponding charge $Q_{i}$. The charge distribution is then projected onto the main axis and two Gaussians are fitted to this distribution. When there are two significant maxima, the showers are mainly from $\pi^{0}$ decay. In the following they are called 'merged showers'. The main background is from single photons that convert in the material just prior to the HPC leading to two showers. However, the magnetic field separates theses clusters in $\phi$, but not in $\theta$. Therefore, merged showers are rejected if the angle in the $\theta-\phi$ plane between the line connecting the two shower centres and the $\theta$ axis is below \pm 100 mrad. This cut removes $60 \%$ of this background.

The invariant mass is calculated from the charge distribution between the two peak positions and the distance between the maxima. Studies using simulation show that both the reconstructed opening angle and the reconstructed energy depend slightly on energy. This is due to the features of the pattern recognition algorithm (maximal size of a shower) and the binning effects in the pad direction, both of which are corrected for. Figure $3 d$ shows the invariant mass for merged showers for energies larger than $10 \mathrm{GeV}$.

The background in the merged shower sample which arises mainly from $\eta$ decays varies with energy: at $6 \mathrm{GeV}, 83 \%$ of the merged showers originate from $\pi^{0}$ s, while at $10 \mathrm{GeV}$ the purity has risen to $90 \%$. For energies greater than $25 \mathrm{GeV}$ the purity is again increasing due to a smaller contamination of $\eta$ 's. The combinatorial background is strongly reduced at these energies, since the algorithm looks only for the closest shower in space in a small cone of less than $2^{0}$ half-opening angle, whereas the average distance to the nearest reconstructible uncorrelated photon is much larger. The detection efficiency of the algorithm is determined by simulation and is shown for the 1992 and 1993 data in figure $5 \mathrm{~d}$. The efficiency is around $10 \%$ at $x_{p}=0.15$ and rises up to $20 \%$ for $x_{p}=0.4$ which is due to the opening angle of the photons which is getting smaller for higher energies. However, for still higher energies the opening angle is too small to resolve two showers, 
hence the efficiency decreases. Due to some changes in the pattern recognition in 1994 some of the merged showers are split into two photons which reduces the efficiency. The tail in figure $3 \mathrm{~d}$ towards larger masses is due mainly to $\pi^{0} \mathrm{~s}$ in which one of the photons converted just before the HPC.

From the simulation it can be deduced that most of the high energy showers found in the HPC are in reality $\pi^{0}$ 's. For high energy $\pi^{0}$ 's the opening angle between the photons is too small to be resolved as two showers in the HPC. Thus, the number of 'all-neutralshowers' made by neutral particles as a function of $x_{p}$ is a useful cross-check. The purity obtained with this method is around $75 \%$ for energies from $6 \mathrm{GeV}$ to $25 \mathrm{GeV}$ and then it drops slowly. The contamination arises mainly from $\eta$ decays as can be seen in the simulation. The efficiency is shown in figure 5 e.

\section{$5 \quad$ Inclusive $\pi^{0}$ cross sections}

As described above $\pi^{0}$ 's can be reconstructed by the following means: pairs of converted photons, pairs of HPC photons, or one converted and one HPC photon, merged showers and all-neutral-showers. For the determination of the cross section only the $\pi^{0}$ reconstruction methods with two converted photons, one converted and one HPC photon and the merged showers are used. The methods with two HPC photons and all showers made by neutral particles were not taken into account because of the background uncertainties: in the first case the background is too large (see figure $3 \mathrm{c}$ ) and in the second case the shower is assumed to originate from a $\pi^{0}$ without any proof. These two methods serve as a cross-check. Using these methods, fits are performed in bins of reconstructed $\pi^{0}$ energy. The signals are fitted by a Gaussian function. In the case of two converted photons the background is parametrised by a straight line in the region $0.09<m_{\gamma \gamma}<0.18 \mathrm{GeV}$. In the cases of one converted and one HPC photon and two HPC photons the signal is wider as shown in figures $3 \mathrm{~b}$ and $3 \mathrm{c}$. Here the background is fitted by the Fermi-function in the range $0.0<m_{\gamma \gamma}<0.4 \mathrm{GeV}: f_{b g}\left(m_{\gamma \gamma}\right)=P_{3} \cdot\left(1 .+P_{4} \cdot\left(m_{\gamma \gamma}-P_{1}\right)\right) /\left(1 .+\exp \left(\frac{P_{1}-m_{\gamma \gamma}}{P_{2}}\right)\right)$, in which $P_{1}, P_{2}, P_{3}$ and $P_{4}$ are free parameters.

For the $\pi^{0}$ 's from merged or neutral showers no combinations with other showers are considered, so there is no combinatorial background. In this case the background is estimated from the simulation (figure $3 \mathrm{~d}$ ).

The cross section is calculated by:

$$
\frac{1 .}{\sigma_{\text {had }}} \frac{d \sigma}{d x_{p}}=\frac{1 .}{N_{\text {had }}} \cdot \frac{N_{\pi^{0}}^{r e c}(D A T A)}{\Delta x_{p}} \cdot \frac{N_{\pi^{0}}^{g e n}(M C)}{N_{\pi^{0}}^{r e c}(M C)}
$$

where $N_{\pi^{0}}^{r e c}\left(N_{\pi^{0}}^{g e n}\right)$ is the number of reconstructed (generated) $\pi^{0}$ s $\mathrm{s}$ in the $x_{p}$ bin. The inverse of the last ratio in this equation represents the efficiency for the $x_{p}$ bin.

The systematic uncertainties in the differential $\pi^{0}$ cross section arise from background parametrisation in the fit procedures, reconstruction efficiencies and corrections of remaining differences between data and simulation such as the material distribution within DELPHI and the energy resolution for converted and HPC photons. The various contributions are summarized in table 1 and are estimated as follows:

- The systematic error of the efficiency is estimated by $(\Delta \epsilon)^{2}=\sum_{i}\left(\Delta \epsilon(i) \frac{N_{\pi}(i)}{N_{\pi, t o t}}\right)^{2}$ where $\Delta \epsilon(i)$ is the error of the efficiency in the $\mathrm{i}$-th $x_{p}$ bin, $N_{\pi}(i)$ is the number of reconstructed $\pi^{0}$ s $\mathrm{s}$ in the i-th $x_{p}$ bin and $N_{\pi, t o t}$ the total number of reconstructed $\pi^{0}$ s. 


\begin{tabular}{|l|c|c|c||}
\hline \multirow{2}{*}{ source of systematic error } & \multicolumn{3}{|c||}{ systematic errors in \% } \\
& 2 conv. $\gamma$ 's & 1 conv./ 1 HPC $\gamma$ & merged showers \\
\hline \hline efficiency & 4.6 & 4.7 & 3.6 \\
additional material & 6.8 & 3.2 & 6.8 \\
only 'best' reconstructed conversions & 2.1 & 3.3 & - \\
additional energy smearing & 2.0 & 3.3 & - \\
additional HPC photons & - & 3.0 & 5.0 \\
background parametrisation & 6.4 & 6.9 & 6.8 \\
cuts in reconstruction algorithms & - & - & 11.8 \\
\hline \hline errors added in quadrature & 10.7 & 10.5 & \\
\hline
\end{tabular}

Table 1: Systematic errors for the number of $\pi^{0}$ s in $\%$.

- As shown in figure 1 the material distribution is not correctly described in the simulation. The radiation length was varied by $4 \%$ for conversions occurring in front of the TPC. A bigger variation results in a shift of the ratio of the measured $\pi^{0}$ cross section obtained with the reconstruction by two converted photons and by one converted photon and one calorimetric photon. The uncertainty in the material distribution also leads to an uncertainty in the number of merged showers. If more photons convert in front of the HPC the number of $\pi^{0}$ 's reconstructed by converted and/or calorimetric photons increases and the number of merged shower decreases. In this case also conversions which occur behind the TPC play a role. For these conversions the uncertainty in the material distribution is estimated to be $7 \%$. This value was obtained by looking at the conversion radius distribution for converted photons where both leptons were seen in the OD and the HPC. Another effect is that more showers with more than two substructures are found in the HPC.

- To determine the error due to the reconstruction algorithms of converted photons only the 'best' measured converted photons (those which were reconstructed by two TPC tracks) are considered and the analysis is repeated. The systematic error represents the difference between the two results.

- To determine the systematic error due to differences in the precision of the energy between data and simulation the precision on the energy is decreased by $0.5 \%$ for converted photons and by $3 \% / \sqrt{E} \oplus 3 \%$ for calorimetric photons.

- The number of HPC photons and merged showers was varied by $3 \%$ to account for differences between data and simulation for HPC showers and the selection criteria to classify photons.

- The systematic error in the background parametrisation for $\pi^{0}$ 's reconstructed by two converted photons was estimated by parametrisation of the background in the invariant mass range range $0.0-0.4 \mathrm{GeV}$ with a Fermi function instead of using a straight line fit. For the combination of one converted and one HPC photon the background was fitted by a third order polynomial instead of a Fermi function. For merged showers the uncertainty was estimated to be $5 \%$.

- Variation of the cuts for the different selection criteria in the reconstruction of merged showers lead to an uncertainty of $6.8 \%$. 


\subsection{Inclusive $\pi^{0}$ production in $q \bar{q}$ events}

The results of the inclusive $\pi^{0}$ cross section measurement using the above methods are shown in figure 7 and reported in table 2.

\begin{tabular}{|c|c|ccr|}
\hline$x_{p}$ & $\left\langle x_{p}\right\rangle$ & $\frac{1}{\sigma_{\text {had }}} \frac{d \sigma}{d x_{p}} \pm \Delta_{\text {stat }} \pm \Delta_{\text {syst }}$ \\
\hline $0.011-0.015$ & 0.013 & $242 . \pm$ & $32 . \pm$ & 26. \\
$0.015-0.020$ & 0.018 & $165 . \pm$ & $16 . \pm$ & 18. \\
$0.020-0.025$ & 0.023 & $140 . \pm$ & $11 . \pm$ & 15. \\
$0.025-0.030$ & 0.027 & $116 . \pm$ & $7 . \pm$ & 12. \\
$0.030-0.035$ & 0.032 & $96.9 \pm$ & $5.7 \pm$ & 10.3 \\
$0.035-0.045$ & 0.040 & $68.7 \pm$ & $2.9 \pm$ & 7.3 \\
$0.045-0.060$ & 0.053 & $45.3 \pm$ & $1.5 \pm$ & 4.8 \\
$0.060-0.080$ & 0.070 & $29.9 \pm$ & $1.4 \pm$ & 3.2 \\
$0.080-0.100$ & 0.090 & $19.4 \pm$ & $0.8 \pm$ & 2.1 \\
$0.100-0.120$ & 0.110 & $14.5 \pm$ & $0.7 \pm$ & 1.5 \\
$0.120-0.145$ & 0.132 & $8.90 \pm$ & $\pm .41 \pm$ & 0.94 \\
$0.145-0.160$ & 0.153 & $7.23 \pm$ & $0.06 \pm$ & 0.85 \\
$0.160-0.175$ & 0.167 & $5.92 \pm$ & $0.06 \pm$ & 0.70 \\
$0.175-0.200$ & 0.188 & $4.49 \pm$ & $0.04 \pm$ & 0.53 \\
$0.200-0.220$ & 0.210 & $3.38 \pm$ & $0.03 \pm$ & 0.40 \\
$0.220-0.240$ & 0.230 & $2.66 \pm$ & $0.03 \pm$ & 0.31 \\
$0.240-0.280$ & 0.260 & $1.86 \pm$ & $0.02 \pm$ & 0.22 \\
$0.280-0.330$ & 0.305 & $1.18 \pm$ & $0.01 \pm$ & 0.14 \\
$0.330-0.380$ & 0.355 & $0.707 \pm 0.009 \pm 0.083$ \\
$0.380-0.440$ & 0.410 & $0.444 \pm 0.007 \pm 0.052$ \\
$0.440-0.500$ & 0.470 & $0.281 \pm 0.006 \pm 0.033$ \\
$0.500-0.570$ & 0.535 & $0.155 \pm 0.004 \pm 0.018$ \\
$0.570-0.650$ & 0.610 & $0.088 \pm 0.003 \pm 0.010$ \\
$0.650-0.750$ & 0.700 & $0.050 \pm 0.002 \pm 0.006$ \\
\hline
\end{tabular}

Table 2: The scale invariant differential cross sections for inclusive $\pi^{0}$ production in multihadronic events at $\sqrt{s}=91.2 \mathrm{GeV}$.

Since the cross section varies by three orders of magnitude over the measured $x_{p}$ region, the ratio of the measured cross sections to the expectation from simulation is shown in figure $7 \mathrm{~b}$ for better visibility. The different methods of $\pi^{0}$ reconstruction lead to consistent results in the regions of overlap. Their weighted mean is used for the final cross section measurement. Integrating over the measured momentum range $\left(0.011<x_{p}<0.75\right)$ gives $\mathrm{N}\left(\pi^{0}\right) / Z_{\text {had }}^{0}=7.1 \pm 0.2$ (stat) \pm 0.8 (syst). Extrapolating into the non-accessible lowenergy range using the shape predicted by the JETSET 7.3 the average number of $\pi^{0}$ 's produced per $Z^{0}$ decay is calculated to be

$$
N\left(\pi^{0}\right) / Z_{\text {had }}^{0}=9.2 \pm 0.2(\text { stat }) \pm 1.0(\text { syst })
$$

The statistical error in the total $\pi^{0}$ rate is dominated by the low energy bins which have large cross sections, but large statistical errors due to the small efficiency for the detection of two converted photons. The systematic error of approximately $11 \%$ has been discussed above in section 5 . 
This result on the number of $\pi^{0}$ 's per hadronic event is in good agreement with the rate predicted by JETSET $7.3(9.95)$ and the measurement at low $x_{p}$ recently published by the L3 Collaboration (see figure 8) of $N\left(\pi^{0}\right) / Z_{\text {had }}^{0}=9.18 \pm 0.03 \pm 0.73$ (9.77 using JETSET 7.3, 8.60 using HERWIG 5.4) [14]. L3 found an important difference in the acceptance calculation between JETSET and HERWIG, due mainly to their strong isolation criterion of the photons from any charged particle tracks $(50 \mathrm{mrad})$. The present analysis is less affected by differences in local particle density because of the good angular resolution of the HPC and the additional use of converted photons which are free of this uncertainty.

Figure 7a shows good agreement between data and JETSET 7.3 for the shape and normalisation of the differential cross section as function of $x_{p}$. The measured spectrum is also compared with other event generators (see figure 9), namely JETSET 7.4, ARIADNE 4.6, and HERWIG 5.8C. Since branching ratios are different in HERWIG compared to reference [2], figure $9 \mathrm{~d}$ shows the results with the branching ratios according to reference [2]. For JETSET 7.4 the default decays are used, which are similar to the decays in reference [2]. Up to $x_{p} \approx 0.5$ the predictions agree with the measured cross section to within about $\pm 10 \%$ for JETSET 7.4 and ARIADNE 4.6. For larger $x_{p}$ more high energy $\pi^{0}$ 's are seen in the simulation. In the case of HERWIG $5.8 \mathrm{C}$ an agreement within $\pm 15 \%$ between the measured and predicted cross section is found.

The measured differential cross section is plotted also as function of $\xi_{p}=\ln \left(1 / x_{p}\right)$. For high $\xi_{p}$ values (i.e. low momenta) the spectrum has a broad Gaussian shape. The peak position is a function of the centre of mass energy and the particle mass as predicted e.g. by the modified leading log approximation (MLLA) [6] which predicts the shape of this distribution for soft gluons. Under the assumption of local parton hadron duality (LPHD) [15] this distribution can be compared directly to the observed hadron spectra. Fitting a Gauss in the range $1.74<\xi_{p}<4.51$ (see figure 10) the maximum of the $\xi_{p}$ distribution is found at:

$$
\xi_{p}^{\star}=3.90_{-0.14}^{+0.24}
$$

with a $\chi^{2}$ of 9.7 with 13 degrees of freedom. This value is in good agreement with the results from other LEP experiments for neutral and charged pions [14,16]. The results are listed in table 3 .

\begin{tabular}{|l|c|l|}
\hline$\xi_{p}^{\star}$ & particle & experiment \\
\hline $3.90_{-0.14}^{+0.24}$ & $\pi^{0}$ & DELPHI \\
$3.96 \pm 0.13$ & $\pi^{0}$ & L3 \\
$3.81 \pm 0.02$ & $\pi^{ \pm}$ & OPAL \\
$3.776 \pm 0.004$ (stat) \pm 0.024 (syst) & $\pi^{ \pm}$ & ALEPH \\
\hline
\end{tabular}

Table 3: Peak position $\xi_{p}^{\star}$ of the $\ln \left(1 / x_{p}\right)$ distribution for neutral and charged pions, for which equal peak positions are expected.

The measured peak position is also in good agreement with the JETSET 7.3 predictions for neutral and charged pions (see figure 10). 


\subsection{Inclusive $\pi^{0}$ production in $b \bar{b}$ events}

The same $\pi^{0}$ reconstruction method was applied to the subset of $Z^{0} \rightarrow b \bar{b}$ events selected with the method described in section 2. Effects due to the $18 \%$ background from light quarks were corrected for by multiplying the data-to-simulation ratio of the number of reconstructed $\pi^{0}$ 's by the number of $\pi^{0}$ 's at the generator level for a pure $b \bar{b}$ sample. The resulting differential cross section is shown in figure $11 \mathrm{a}$ and listed in table 4.

\begin{tabular}{|c|c|ccr|}
\hline$x_{p}$ & $\left\langle x_{p}\right\rangle$ & \multicolumn{3}{|c|}{$\frac{1}{\sigma_{b \bar{b}}} \frac{d \sigma}{d x_{p}} \pm \Delta_{\text {stat }} \pm \Delta_{\text {syst }}$} \\
\hline $0.011-0.025$ & 0.018 & $175 . \pm$ & $36 . \pm$ & 19. \\
$0.025-0.045$ & 0.035 & $106 . \pm$ & $7.8 \pm$ & 11. \\
$0.045-0.060$ & 0.053 & $52.5 \pm$ & $4.9 \pm$ & 5.6 \\
$0.060-0.080$ & 0.070 & $29.6 \pm$ & $2.6 \pm$ & 3.4 \\
$0.080-0.100$ & 0.090 & $18.5 \pm$ & $2.0 \pm$ & 2.0 \\
$0.100-0.145$ & 0.123 & $12.2 \pm$ & $1.0 \pm$ & 1.2 \\
$0.145-0.160$ & 0.153 & $6.67 \pm$ & $0.91 \pm$ & 0.79 \\
$0.160-0.175$ & 0.167 & $4.90 \pm$ & $0.67 \pm$ & 0.58 \\
$0.175-0.200$ & 0.188 & $3.23 \pm$ & $0.45 \pm$ & 0.38 \\
$0.200-0.220$ & 0.210 & $2.37 \pm$ & $0.33 \pm$ & 0.28 \\
$0.220-0.240$ & 0.230 & $1.79 \pm$ & $0.25 \pm$ & 0.21 \\
$0.240-0.280$ & 0.260 & $1.18 \pm$ & $0.16 \pm$ & 0.14 \\
$0.280-0.330$ & 0.305 & $0.646 \pm$ & $0.089 \pm$ & 0.076 \\
$0.330-0.380$ & 0.355 & $0.328 \pm$ & $0.045 \pm$ & 0.039 \\
$0.380-0.440$ & 0.410 & $0.211 \pm$ & $0.029 \pm$ & 0.025 \\
$0.440-0.500$ & 0.470 & $0.101 \pm$ & $0.014 \pm$ & 0.012 \\
$0.500-0.570$ & 0.535 & $0.0382 \pm 0.0051 \pm 0.0045$ \\
$0.570-0.650$ & 0.610 & $0.0211 \pm 0.0034 \pm 0.0025$ \\
\hline
\end{tabular}

Table 4: The scale invariant differential cross sections for inclusive $\pi^{0}$ production in b-events at $\sqrt{s}=91.2 \mathrm{GeV}$.

For better visibility the ratio of the measured cross sections to the expectation from simulation is shown in figure $11 \mathrm{~b}$. Compared with $q \bar{q}$ events the spectrum is softer due to the higher multiplicity in $\mathrm{B}$ hadron decays. Integrating the number of $\pi^{0}$ 's in the scaled momentum range between 0.025 and 0.57 gives $\mathrm{N}\left(\pi^{0}\right) / b \bar{b}=4.9 \pm 0.2$ (stat) \pm 0.5 (syst). The rest of the spectrum was extrapolated using the shape predicted by JETSET 7.3. The average number of neutral pions is found to be:

$$
\mathrm{N}\left(\pi^{0}\right) / b \bar{b}=10.1 \pm 0.4(\text { stat }) \pm 1.1 \text { (syst) }
$$

which is also in good agreement with the JETSET 7.3 prediction of 10.85 neutral pions per event.

As in the case of $q \bar{q}$ events the systematic error is $11 \%$, compared to which the error due to impurities in the b-tagged sample can be neglected. In figure 12 the measured spectrum is compared with JETSET 7.4, ARIADNE 4.6, and HERWIG 5.8C. JETSET and ARIADNE both use Peterson fragmentation for the decay of heavy quarks. For HERWIG the expectation with default branching ratios and the branching ratios from [2] are shown. For JETSET 7.4 the default decays were taken which are similar to the decays from reference [2]. 
All models with the exception of HERWIG 5.8C with default branching ratios show agreement to within $\pm 20 \%$ with the differential cross sections in both the shape and normalisation. In the case of HERWIG $5.8 \mathrm{C}$ with default branching ratios there is a mismatch at high $x_{p}\left(x_{p}>0.3\right)$ which arises from differences in the branching ratios used in HERWIG and those quoted in reference [2], as deduced from figures $12 \mathrm{c}$ and $12 \mathrm{~d}$.

In figure 13 the ratio $\mathrm{R}$ of the measured differential $\pi^{0}$ cross section for $b \bar{b}$ events to $q \bar{q}$ events is shown. The different fragmentation of $b \bar{b}$ events is evident: at higher $x_{p}$ the $\pi^{0}$ production is almost a factor of two lower for $b \bar{b}$ events compared to $q \bar{q}$ events. Using the ratio has the advantage that it is not affected by the overall normalisation. Comparing this ratio with the prediction of the JETSET 7.3 generator for neutral and charged pions, an agreement within $\pm 20 \%$ is found. The ratio of the number of $\pi^{0}$ 's in $b \bar{b}$ events to hadronic events in the $x_{p}$ range $0.025-0.57$ is measured to be $1.07 \pm$ $0.04 \mathrm{mbox}($ stat $) \pm 0.01 \mathrm{mbox}\left(\right.$ syst). Extrapolating to the non-accessible $x_{p}$ region using the shape predicted by JETSET 7.3 gives:

$$
\frac{\mathrm{N}_{\pi^{0}}(b \bar{b})}{\mathrm{N}_{\pi^{0}}\left(Z^{0}\right)}=1.09 \pm 0.05(\text { stat }) \pm 0.01 \text { (syst) }
$$

which is in good agreement with the predicted value of 1.09 from the JETSET 7.3 generator. The systematic error arises from the contamination of non- $b \bar{b}$ events in the b-tagged sample.

To determine the average $\pi^{0}$ multiplicity in the decay of primary B-hadrons, the technique described in reference [17] was used. Using the simulation for detector effects, and for the contamination from non- $b \bar{b}$ events, the rapidity distribution $r(|y|)$ (with respect to the thrust axis of the event) of $\pi^{0}$ 's is corrected bin by bin giving the rapidity distributions for $b \bar{b}$ events which is shown in figure 14 . The distribution is then fitted to the expression

$$
r(|y|)=N\left[(1-\alpha) f_{f}(|y|)+\alpha f_{d}(|y|)\right]
$$

where $f_{f}$ and $f_{d}$ are the distributions expected from fragmentation and decays of $\mathrm{B}$ hadrons respectively, $\alpha$ is the fraction of particles arising from the decay of the $\mathrm{B}$ hadron, and $N$ is a normalisation factor. Taking $f_{f}$ and $f_{d}$ from the JETSET PS model with Peterson fragmentation gives $\alpha=0.55 \pm 0.02$, with a $\chi^{2}$ of 14.0 with 11 degrees of freedom. The result of the fit from this model was used for the measurement, because it was shown to reproduce the inclusive distributions reasonably well [17]. By taking the distributions $f_{f}$ and $f_{d}$ from:

- HERWIG 5.7, with cluster fragmentation;

- JETSET 7.4 PS, with string fragmentation and Bowler parameterisation of the fragmentation function;

- JETSET 7.3 PS with Lund symmetric fragmentation function;

- ARIADNE 4.6, with dipole fragmentation

different results on the fraction $\alpha$ of particles coming from the $\mathrm{B}$ hadron decay are obtained. The RMS spread of these measurement is used as an estimate of the systematic error from this source. A second source of systematic error includes the effect of an additional uncertainty of $\pm 15 \%$ (uncorrelated) on the efficiency for each rapidity bin to account for possible rapidity-dependent effects. By folding these results with equation (6), the average number of $\pi^{0}$ 's from the decay of a $\mathrm{B}$ hadron is found to be:

$$
\mathrm{N}\left(B \rightarrow \pi^{0} X\right) / \mathrm{B} \text { hadron }=2.78 \pm 0.15(\text { stat }) \pm 0.60 \text { (syst) }
$$




\section{Conclusions}

The inclusive $\pi^{0}$ cross section in $Z^{0} \rightarrow q \bar{q}$ and $Z^{0} \rightarrow b \bar{b}$ events in the momentum range $x_{p}=0.011$ to 0.75 is measured using the DELPHI detector at LEP I. Five different reconstruction methods are used and they give consistent results in the regions of overlap. The total number of $\pi^{0}$ 's in $Z^{0} \rightarrow q \bar{q}$ decays is $9.2 \pm 0.2$ (stat) \pm 1.0 (syst), in agreement with the prediction of 9.95 by JETSET 7.3 with the tuning described in [1]. Various fragmentation models agree with the differential cross section to within $10 \%$ for $x_{p}$ below 0.4 . For higher $x_{p}$ the shape is not modelled well. The cross section plotted as a function of $\xi_{p}=1 / x_{p}$ is assumed to be Gaussian shaped near the maximum which is at $\xi_{p}^{\star}=3.90_{-0.14}^{+0.24}$, in agreement with the results from other LEP experiments for neutral and charged pions. In $Z^{0} \rightarrow b \bar{b}$ events the average number of $\pi^{0}$ s is $\mathrm{N}\left(\pi^{0}\right) / b \bar{b}=10.1 \pm 0.4$ (stat) \pm 1.1 (syst). The ratio of the number of $\pi^{0}$ s in $b \bar{b}$ events to hadronic $Z^{0}$ events, which is less affected by the normalisation error, is $1.09 \pm 0.05$ (stat) \pm 0.01 (syst), in good agreement with the value of 1.09 predicted by the JETSET 7.3 generator. An average $\pi^{0}$ multiplicity from the $\mathrm{B}$ hadron decay of $\mathrm{N}\left(B \rightarrow \pi^{0} X\right) / \mathrm{B}$ hadron $=2.78 \pm 0.15$ (stat) \pm 0.60 (syst) is found.

\section{Acknowledgements}

We are greatly indebted to our technical collaborators and to the funding agencies for their support in building and operating the DELPHI detector, and to the members of the CERN-SL Division for the excellent performance of the LEP collider. 


\section{References}

[1] W. de Boer, H. Fürstenau, Comparison of DELPHI data with QCD models, DELPHI Note 91-75 Phys 129;

K. Hamacher, M. Weierstall, The next round of Hadronic Generator Tuning heavily based on identified particle Data, preprint Univ. Wuppertal WU B 9507.

[2] Particle Data Group, Phys. Rev. D50 (1994).

[3] T. Sjöstrand, Comp. Phys. Comm. 82 (1994) 74;

T. Sjöstrand, Comp. Phys. Comm. 39 (1986) 347.

[4] L. Lönnblad, DESY 92-046.

[5] G. Marchesini and B.R. Webber, Nucl. Phys. B238 (1984) 1.;

G. Marchesini et al., Comp. Phys. Comm. 67 (1992) 465;

T. Sjöstrand and M. Bengtsson, Comp. Phys. Comm. 43 (1987) 367.

[6] Y.I. Azimov, Yu.L. Dokshitzer, V.A. Khoze and S.I. Troyan, Z. Phys. C27 (1985) 65, and C31 (1986) 213;

C.P. Fong, B.R. Webber, Phys. Lett. B229 (1989) 289.

[7] DELPHI collaboration, P. Abreu et al., Nucl. Instr. Meth. A323 (1992) 351.

[8] H.G. Fischer and O. Ullaland, IEEE Trans. Nucl. Sci. NS-27 (1980) 38;

M. Berggren et al., Nucl. Instr. and Meth. A225 (1984) 477;

A.Cattai et al., Nucl. Instr. and Meth. A235 (1985) 310;

V. Gracco et. al., Nucl. Instr. and Meth. A252 (1986) 572;

F.L. Navarria et al., Nucl. Instr. and Meth. A257 (1987) 499;

H.G. Fischer et al., Nucl. Instr. and Meth. A265 (1988) 218;

A. Algeri et al., CERN-PPE/95-04 (1995).

[9] Ch. Kreuter, Longitudinal Shower Development in the DELPHI Electromagnetic Calorimeter HPC, Preprint Univ. Karlsruhe IEKP-KA/93-9.

[10] DELPHI collaboration, P. Abreu et al., Z. Phys. C54 (1992) 55.

[11] DELPHI collaboration, DELSIM User's Guide, DELPHI Note 89-67 PROG 142.

[12] ALEPH collaboration, D. Buskulic et al., Phys. Lett. B313 (1993) 535;

DELPHI collaboration, P. Abreu et al., Z. Phys. C65 (1995) 555

[13] R.L. Ford, W.R. Nelson, The EGS code system, Preprint SLAC-210 (1978).

[14] L3 collaboration, M. Acciarri et al., Phys. Lett B328 (1994) 223.

[15] D. Amati and G. Veneziano, Phys. Lett. B83 (1979) 87; Y.I. Azimov et al., Phys. Lett. B165 (1985) 147.

[16] OPAL collaboration, R. Akers et al., Z. Phys. C63 (1994) 181 ; ALEPH collaboration, D. Buskulic et al., Z. Phys. C66 (1995) 355.

[17] DELPHI collaboration, P. Abreu et al., Phys. Lett. B347 (1995) 447. 


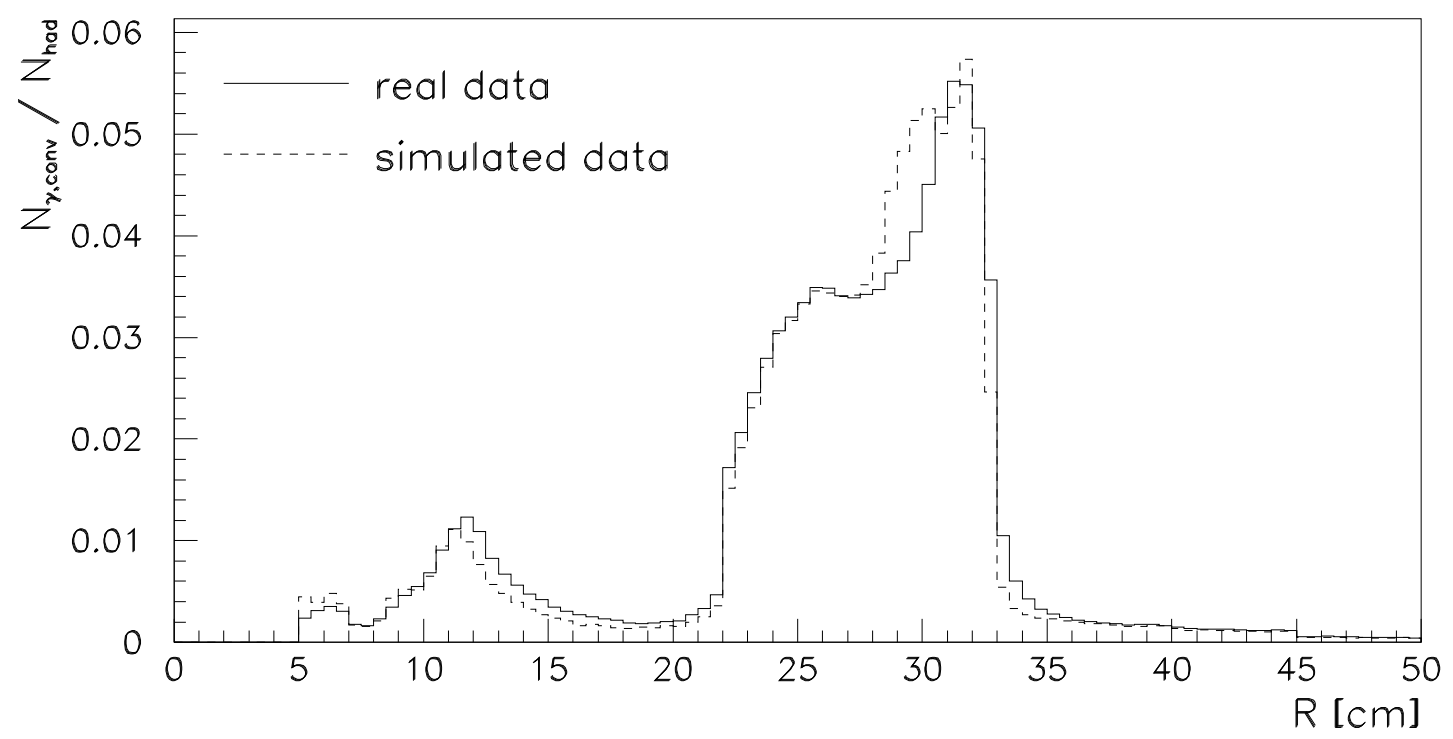

Figure 1: Distribution of conversion radii reconstructed in the TPC for real and simulated data. The number of conversions is normalised to the number of events.

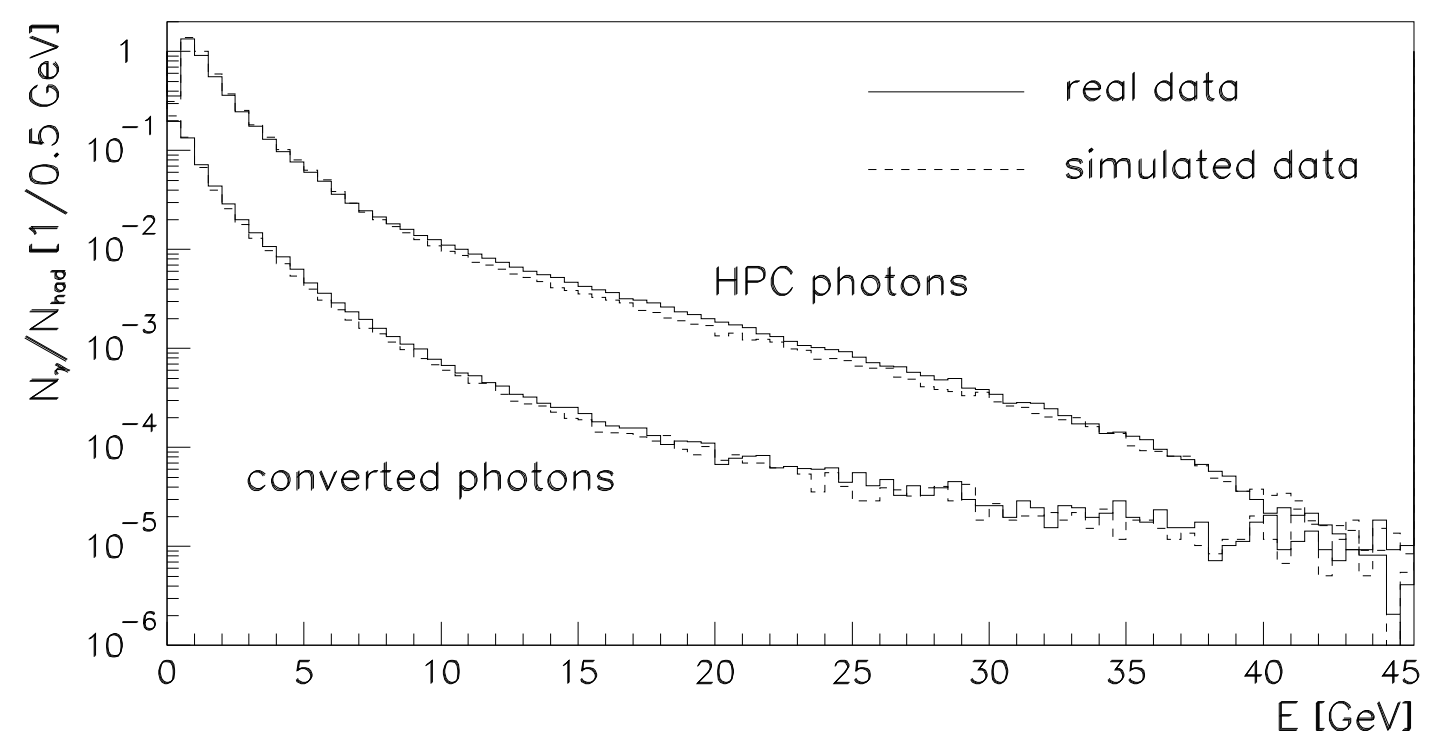

Figure 2: Comparison of the energy spectra of converted and calorimetric photons for real and simulated data. The number of photons is normalised to the number of events. 

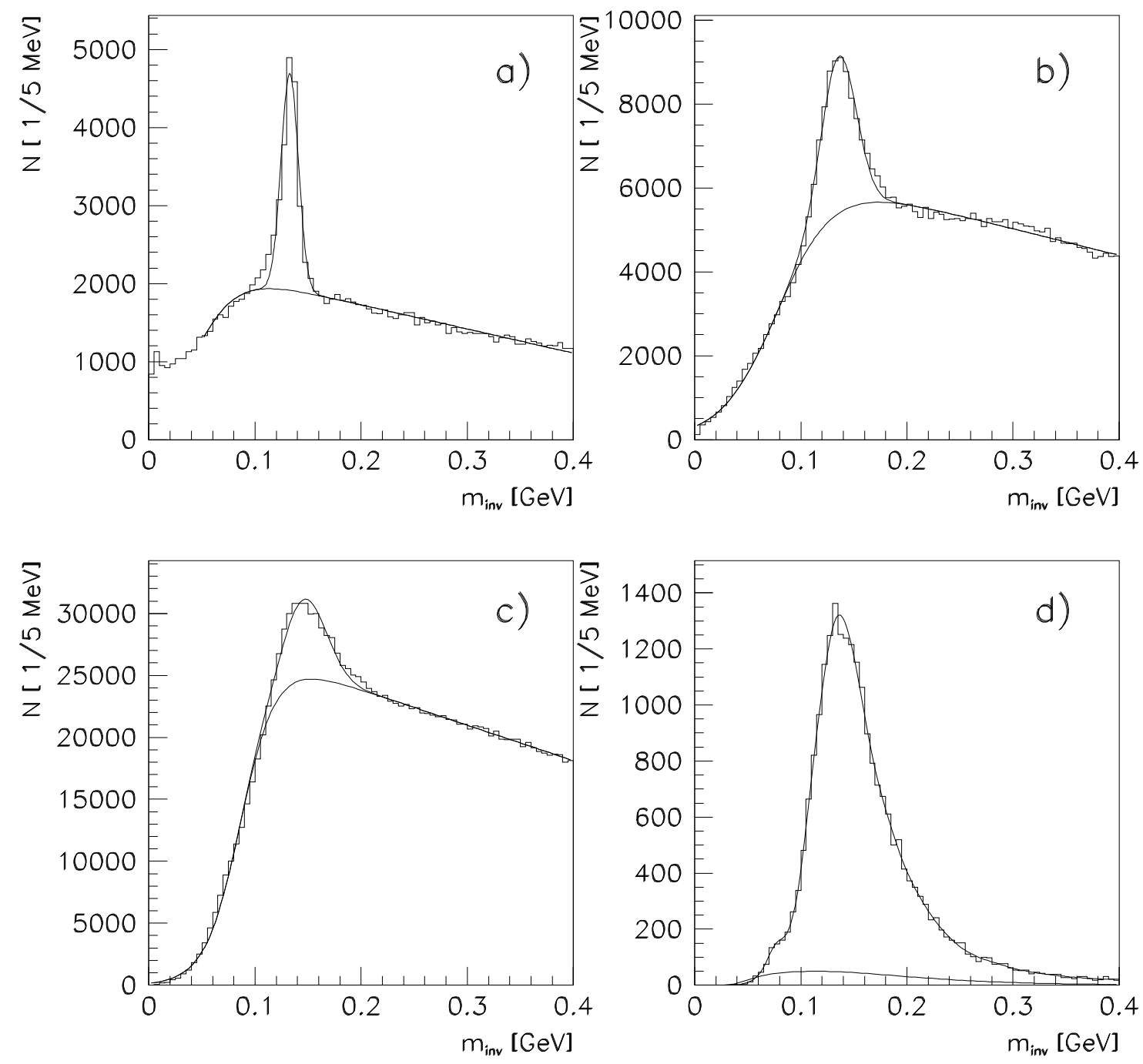

Figure 3: Invariant mass spectra data from 1992 - 1994 for the four different $\pi^{0}$ reconstruction methods: a) Combination of two converted photons; b) Combination of a converted photon with an HPC photon; c) Combination of two HPC photons in the energy range from 4 to $8 \mathrm{GeV}$; d) Mass calculated from merged HPC showers in the energy range $10-25 \mathrm{GeV}$; due to changes in the HPC pattern recognition only the spectrum for 1992 and 1993 data is shown. In $(\mathrm{a}, \mathrm{b}, \mathrm{c})$ the solid curves represent the fit results; in (d) the background could not be determined from data and was taken from the simulation. 

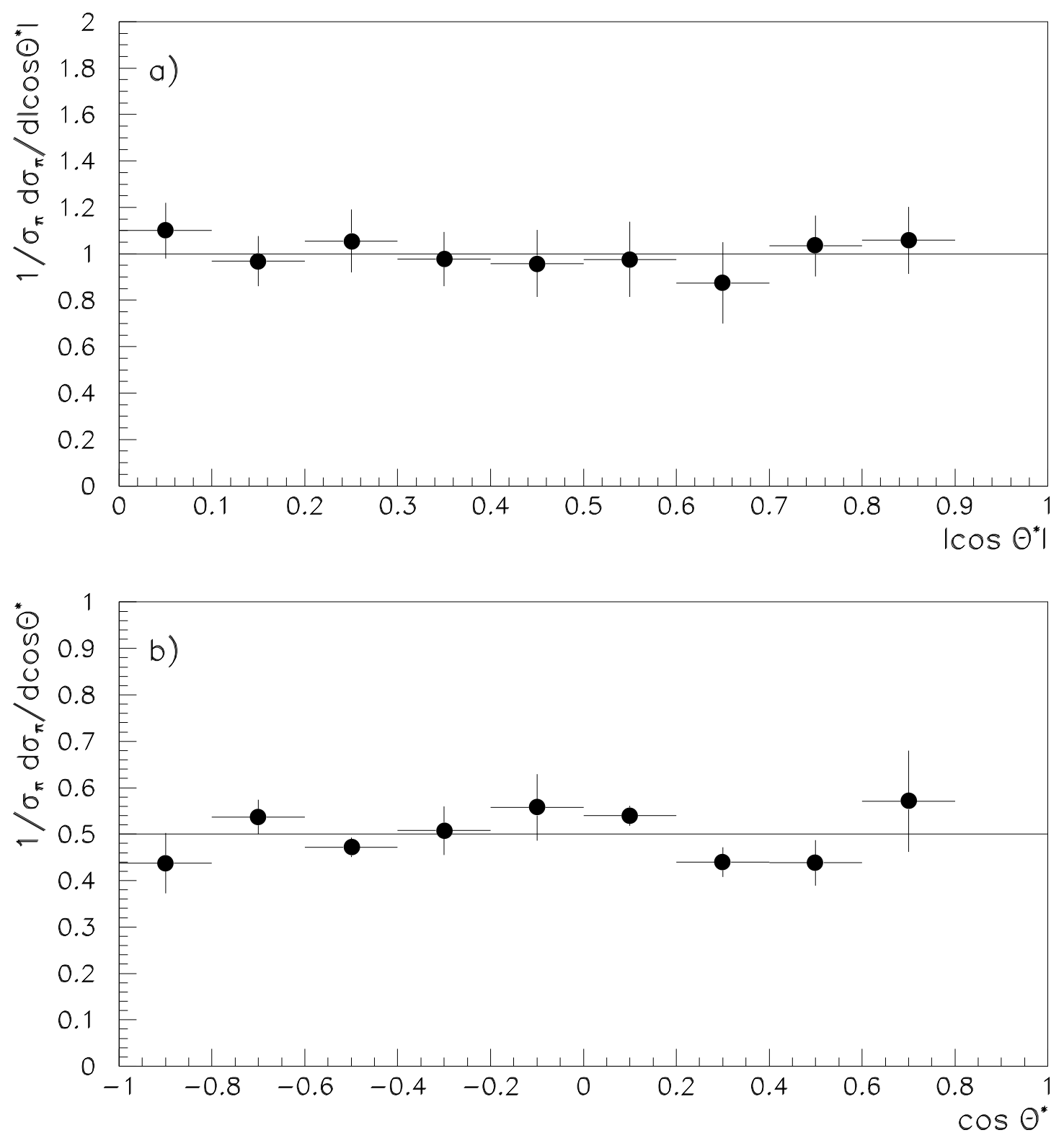

Figure 4: Decay angular distribution for photons coming from $\pi^{0}$ 's in the $\pi^{0}$ rest frame for $\pi^{0}$ 's reconstructed by (a) two converted photons and (b) one converted and one calorimetric photon. In the second case, only the angle of the converted photon is shown. 

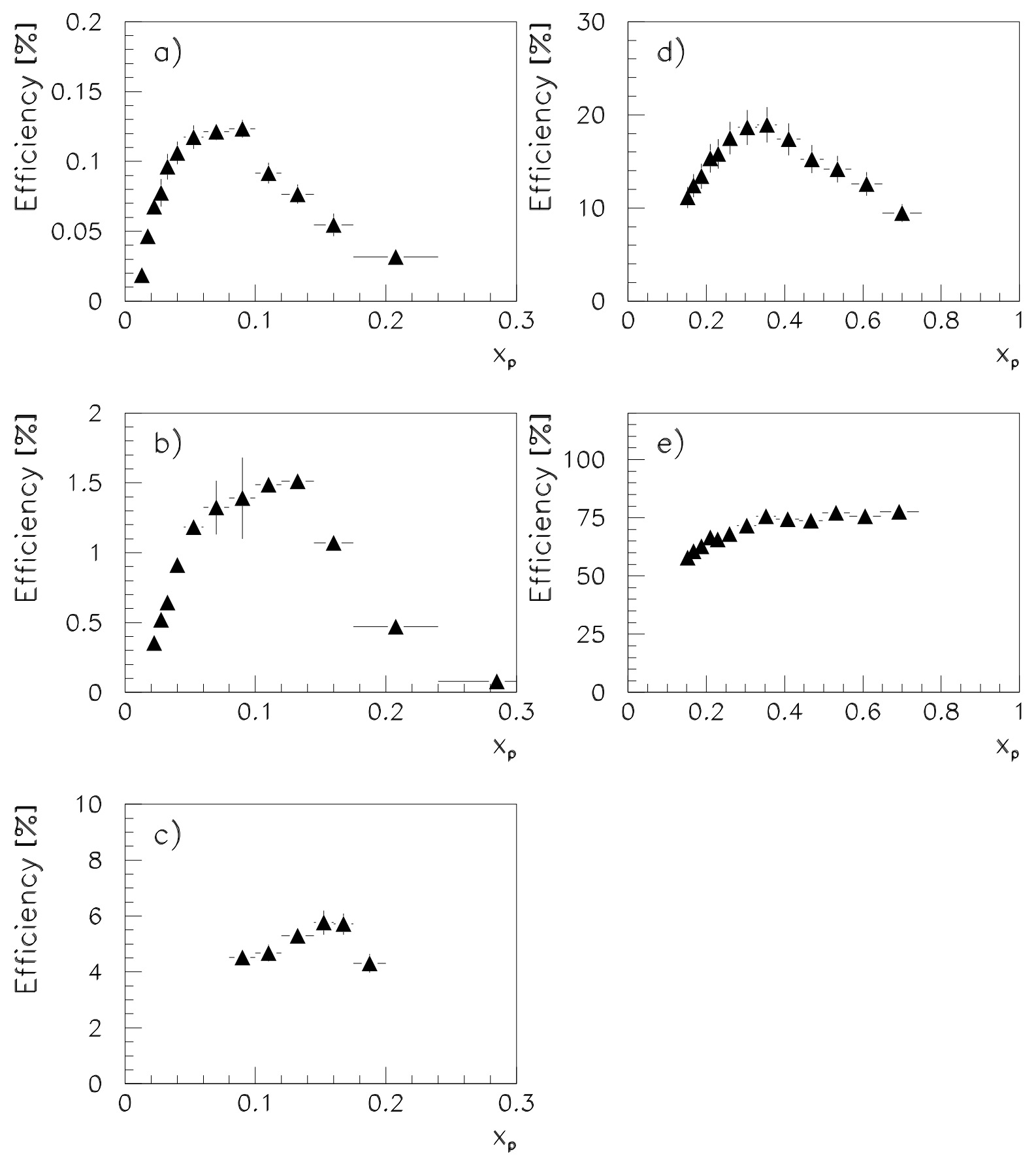

Figure 5: Efficiencies determined from simulation for the five different $\pi^{0}$ reconstruction methods for 1992 and 1993 data: a) combination of two converted photons; b) combination of a converted photon with a HPC photon; c) combination of two HPC photons; d) merged HPC showers; e) Sum of all showers found in the HPC which were not linked to a charged particle. 

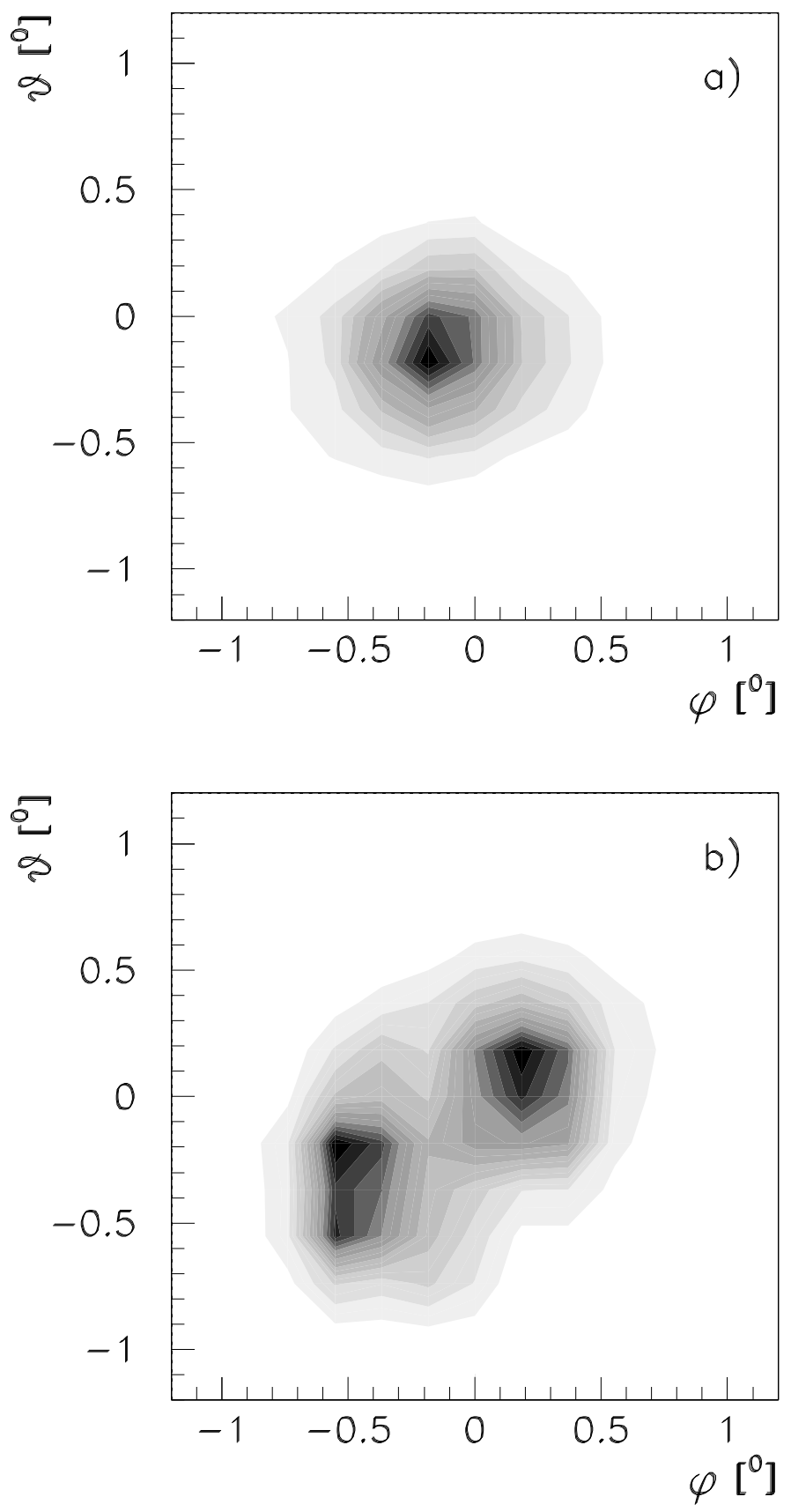

Figure 6: Lateral charge distribution in the $\Delta \vartheta-\Delta \varphi$ plane from the centre of gravity of a) a $24.5 \mathrm{GeV}$ single photon from a $Z^{0} \rightarrow \mu \mu \gamma$ event, b) a $28.9 \mathrm{GeV} \pi^{0}$ from a $\tau^{-} \rightarrow \nu \rho^{-} \rightarrow \nu \pi^{-} \pi^{0}$ decay. 

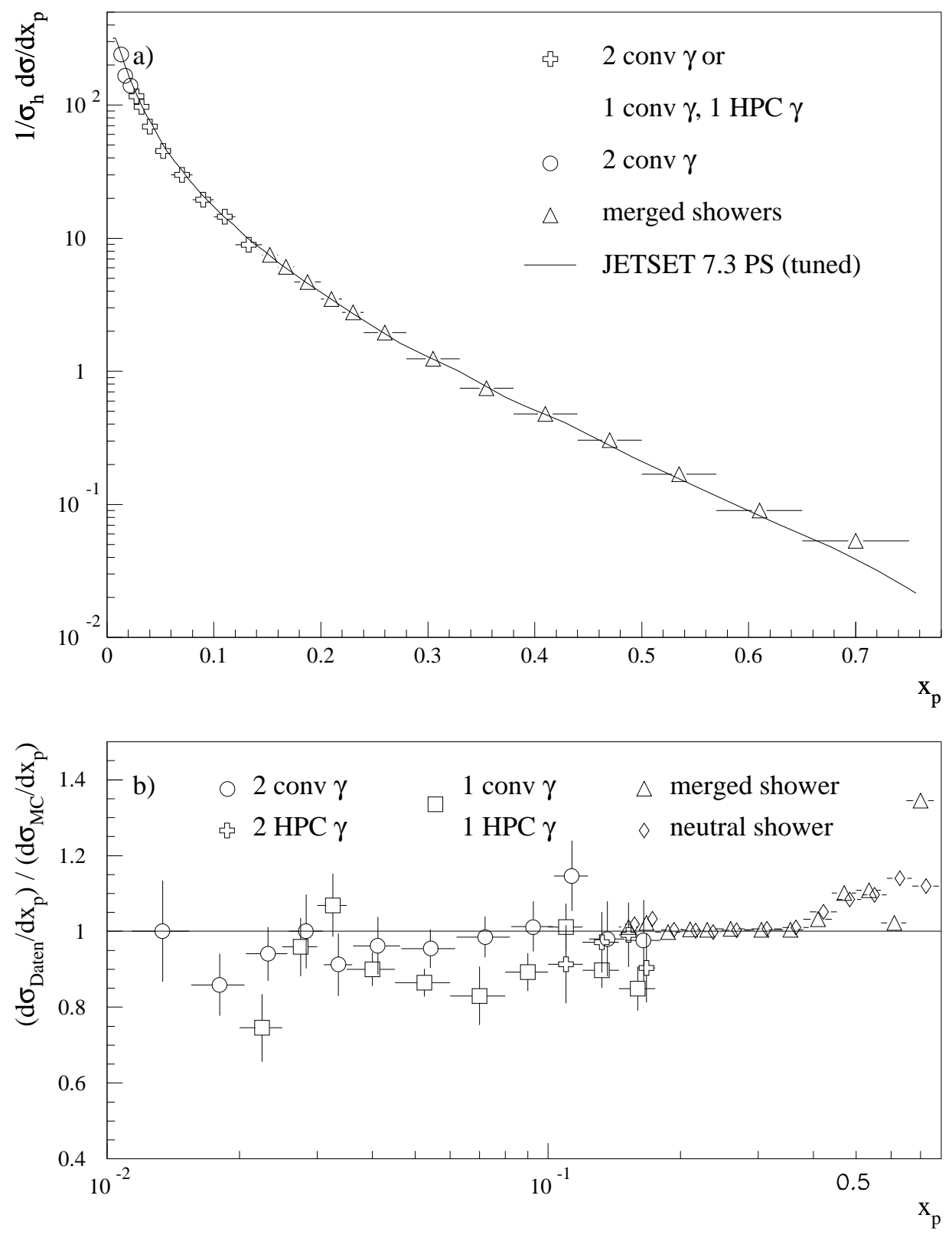

Figure 7: a) Differential inclusive $\pi^{0}$ cross section in $Z^{0} \rightarrow q \bar{q}$ events normalised to the total hadronic cross section, $1 / \sigma_{h} d \sigma / d x_{p}$. The points represent the measured cross section. Only statistical errors are shown. The line shows the JETSET 7.3 prediction using DELPHI tuning. b) Comparison of the results of the different $\pi^{0}$ reconstruction methods with JETSET 7.3 prediction. Here $x_{p}$ is plotted on a logarithmic scale. Besides the methods used for the determination of the cross section, the $\pi^{0}$ reconstruction methods from two HPC photons and all showers not linked to charged particles are also shown for consistency checks. 

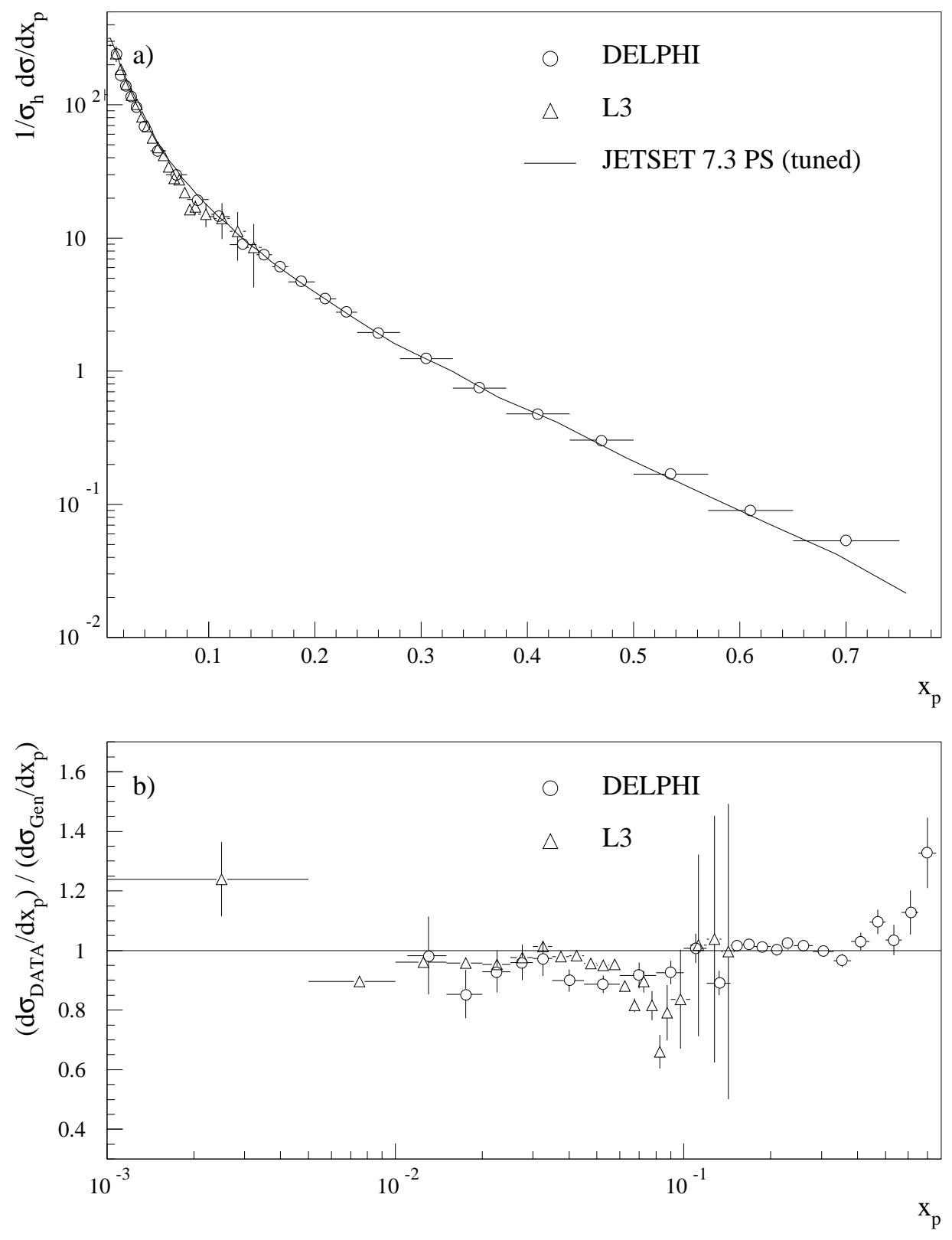

Figure 8: a) Measured differential cross section as function of $x_{p}$ in $Z^{0} \rightarrow q \bar{q}$ events from DELPHI and L3. b) Ratio of the measured differential cross section to the cross section predicted by the JETSET 7.3 event generator. Here $x_{p}$ is plotted on a logarithmic scale. Only statistical errors are shown. 

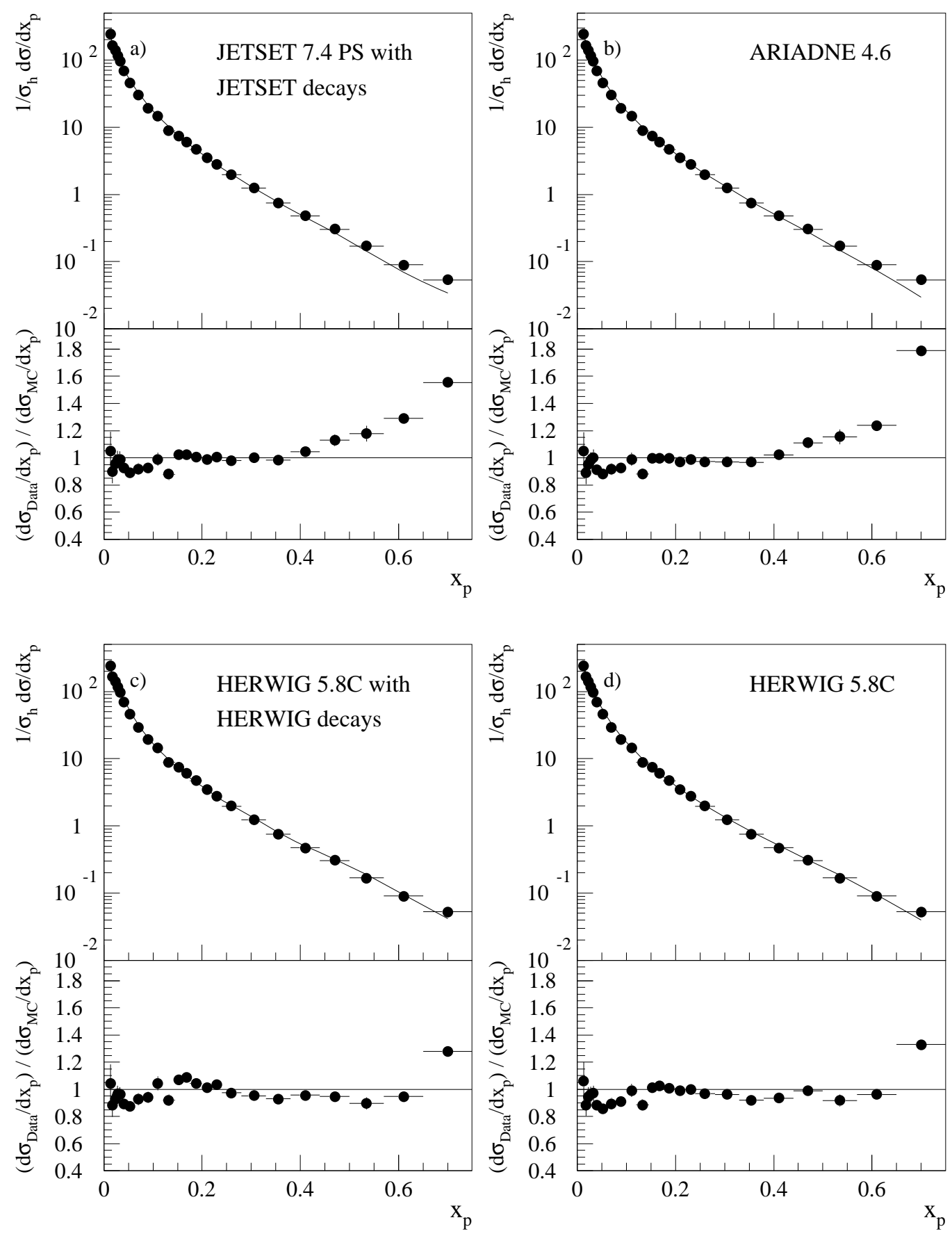

Figure 9: Comparison of the measured differential cross section as function of $x_{p}$ in $Z^{0} \rightarrow q \bar{q}$ events with different models, namely (a) JETSET 7.4, (b) ARIADNE 4.6, (c) HERWIG 5.8C and (d) HERWIG 5.8C with the same branching ratios as in reference [2]. The ratio of measured to predicted differential cross section is below each plot. Only statistical errors are shown. 


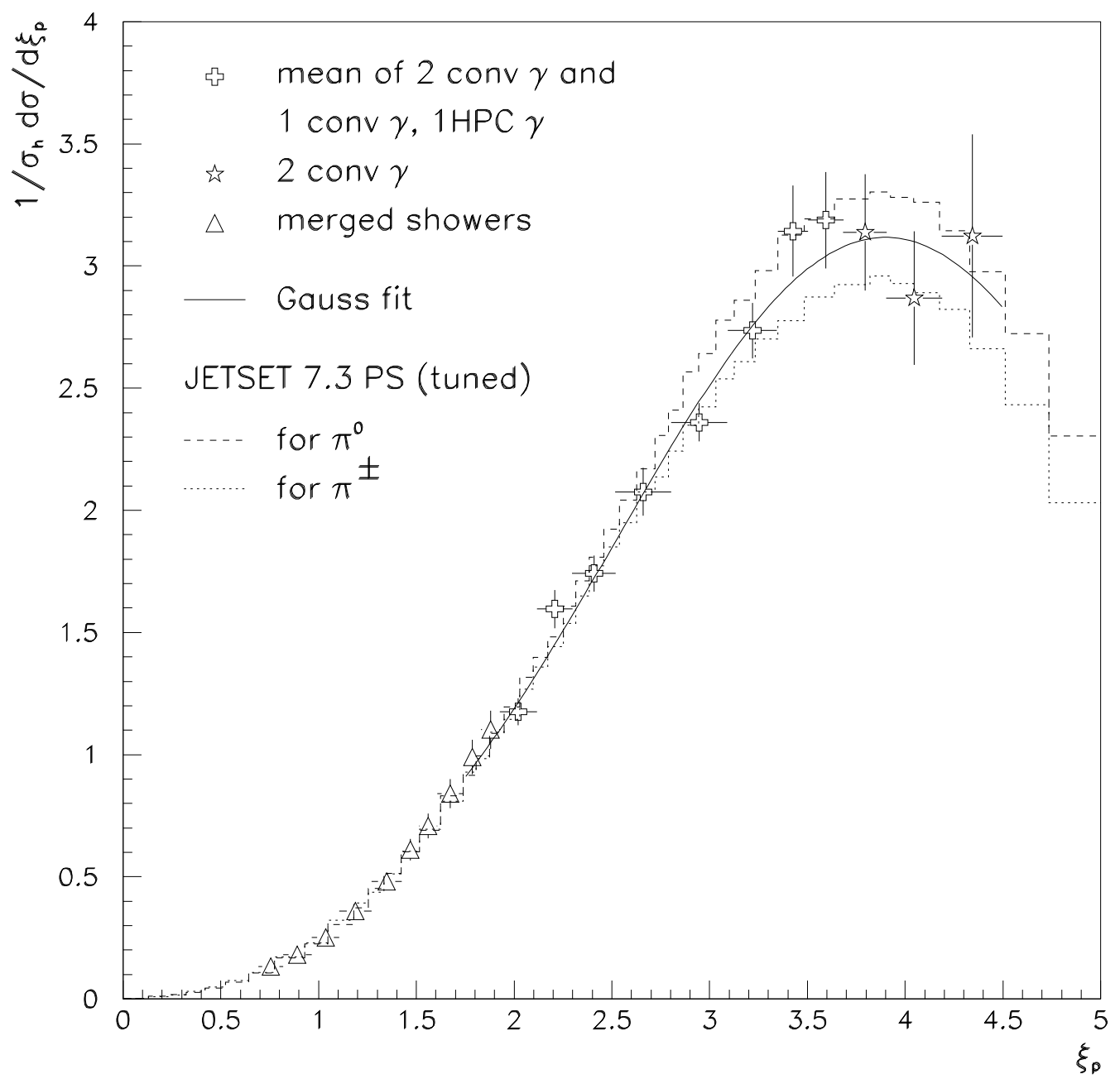

Figure 10: The inclusive $\xi_{p}$ spectra normalised to the total hadronic cross section. Only statistical errors are shown. The curve shows the fit result within the fitted region. The dashed lines show the prediction from the JETSET 7.3 generator for neutral pions and charged pions. 

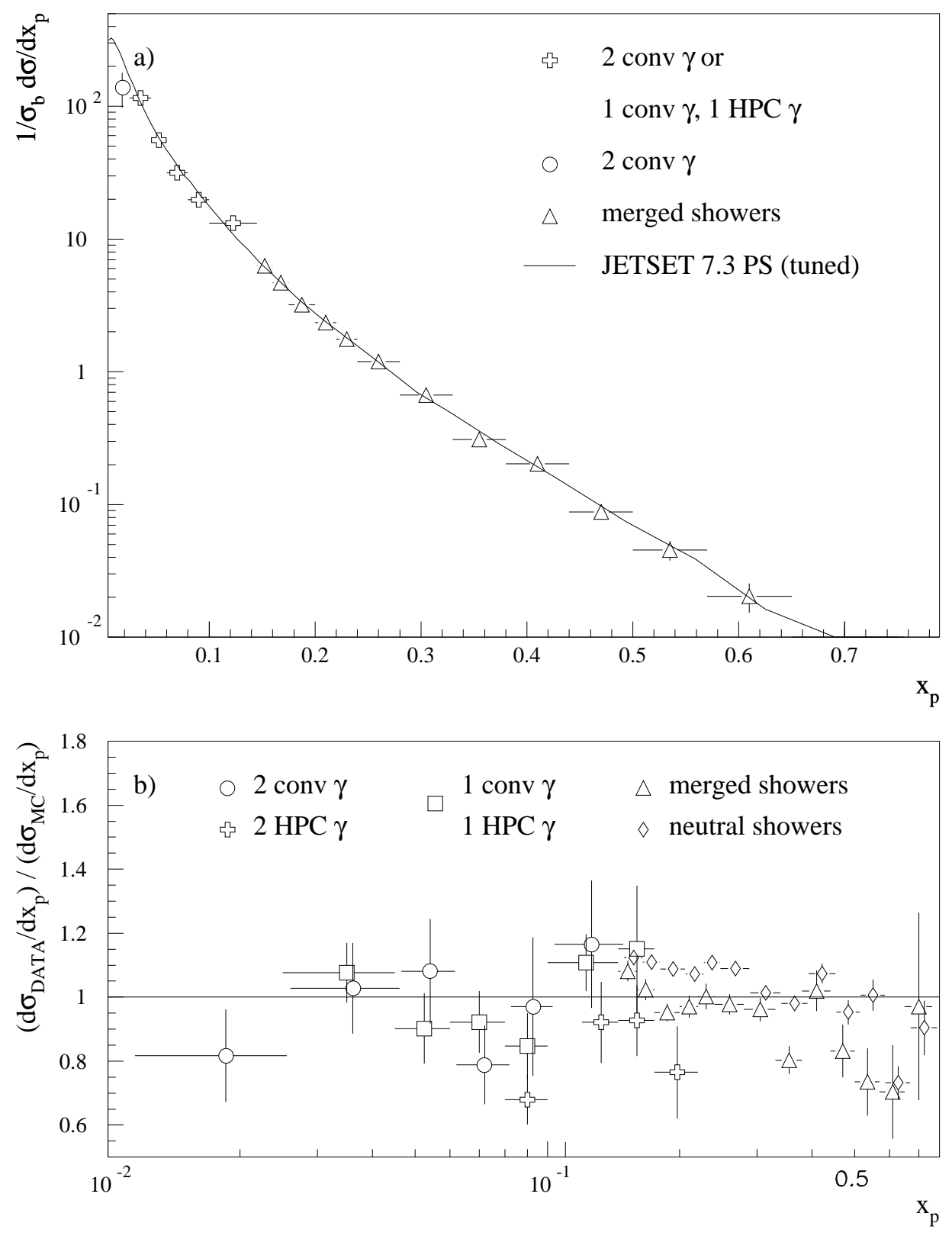

Figure 11: a) Differential inclusive $\pi^{0}$ cross section in $Z^{0} \rightarrow b \bar{b}$ events normalised to the total $b \bar{b}$ cross section, $1 / \sigma_{b \bar{b}} d \sigma / d x_{p}$. The points represent the measured cross section. Only statistical errors are shown. The line shows the JETSET 7.3 prediction using DELPHI tuning. b) Comparison of the results of the different $\pi^{0}$ reconstruction methods with the JETSET 7.3 prediction. Here $x_{p}$ is plotted on a logarithmic scale. Besides the methods used for the determination of the cross section, the $\pi^{0}$ reconstruction methods from two HPC photons and all showers not linked to charged particles are also shown for consistency checks. 

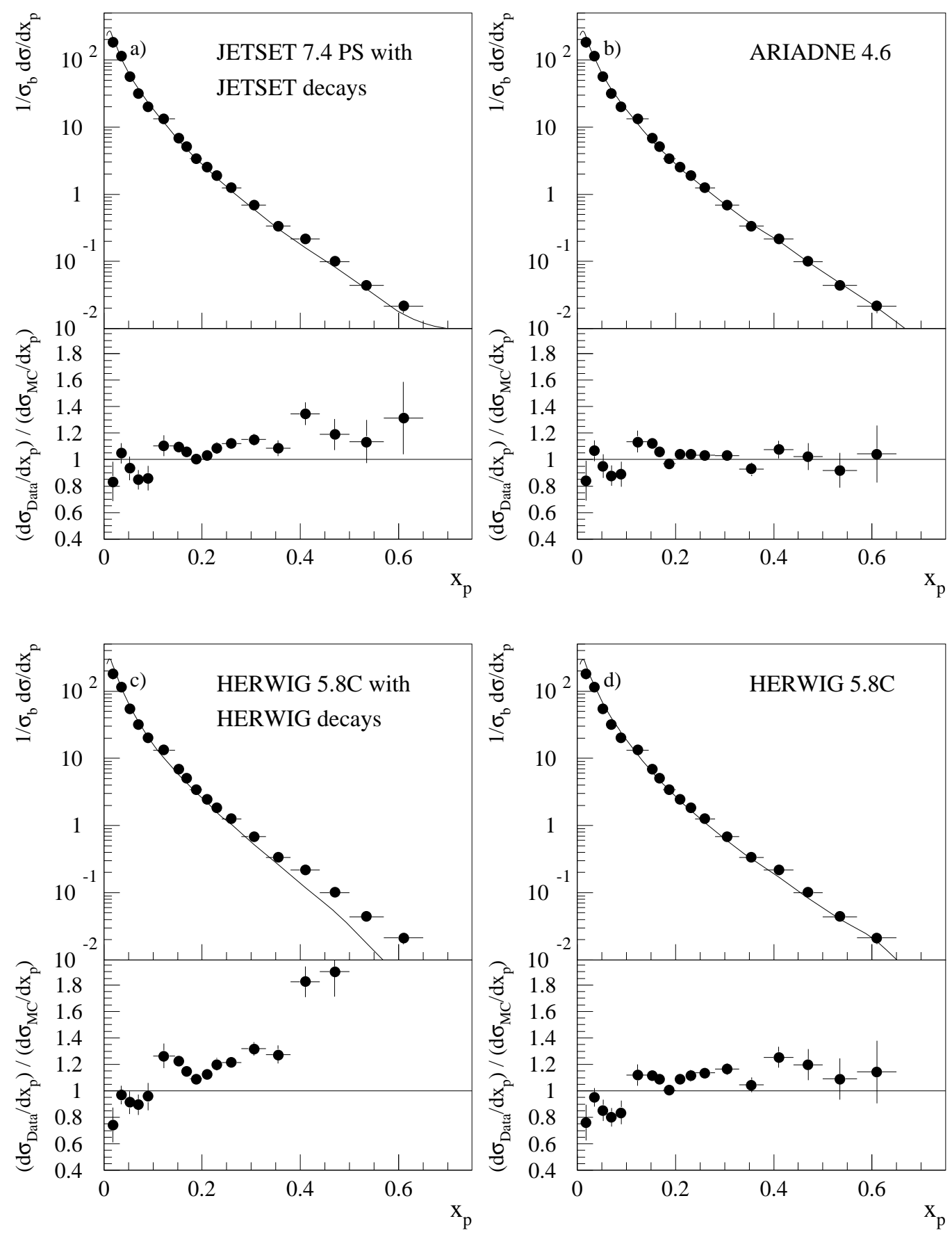

Figure 12: Comparison of the measured differential cross section as function of $x_{p}$ in $Z^{0} \rightarrow b \bar{b}$ events with different MC event generators, namely (a) JETSET 7.4, (b) ARIADNE 4.6, (c) HERWIG 5.8C and (d) HERWIG 5.8C with the same branching ratios as in reference [2]. The ratio of measured to predicted differential cross section is plotted below each plot. Only statistical errors are shown. 

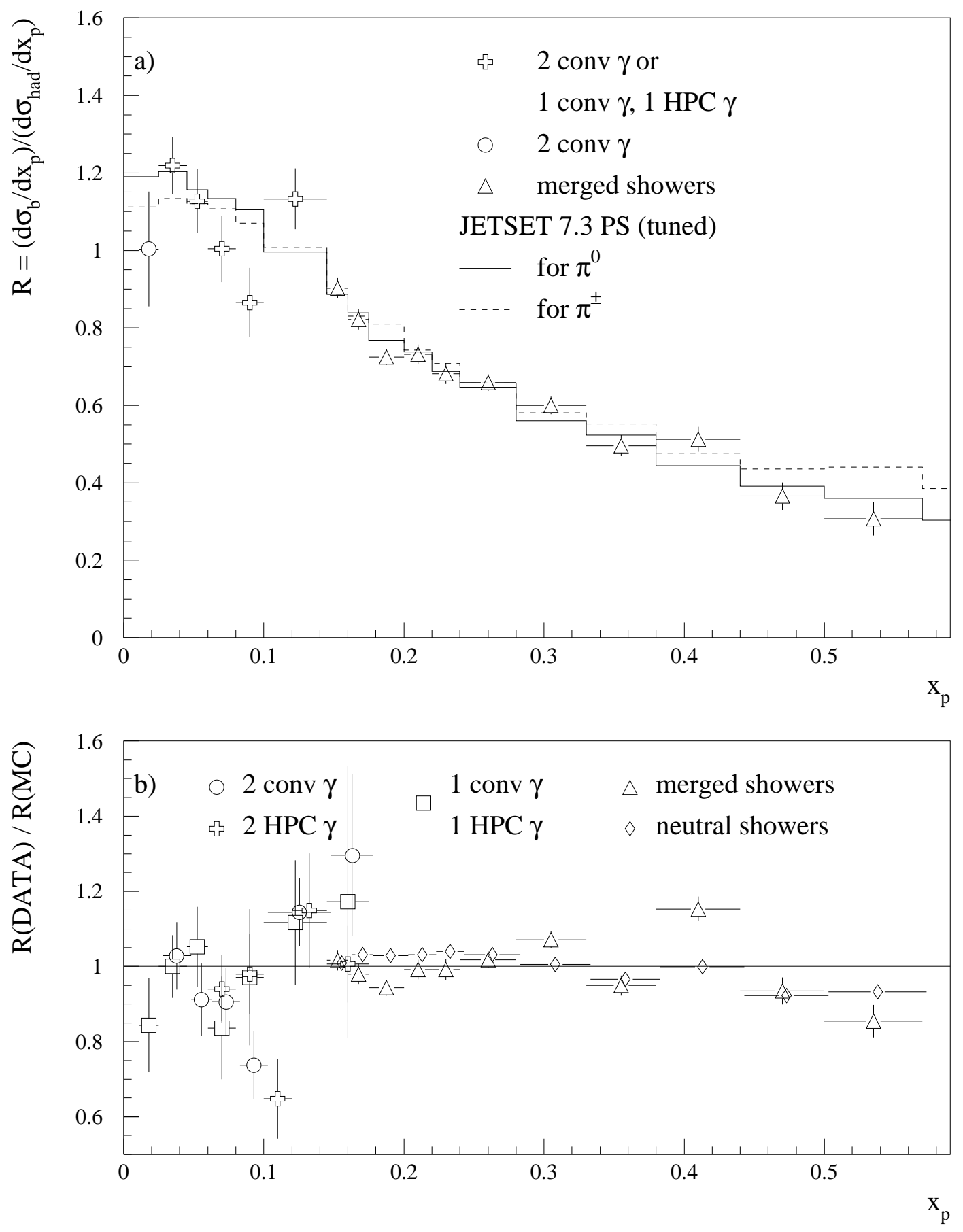

Figure 13: (a) Ratio $\mathrm{R}$ of the measured differential cross section of $b \bar{b}$ events and hadronic events as function of $x_{p}$. The lines show the prediction of the JETSET 7.3 model for neutral and charged pions. (b) Measured ratio divided by the predicted ratio from the JETSET 7.3 model. Besides the methods used for the determination of the ratio $\mathrm{R}$ the $\pi^{0}$ reconstruction methods from two HPC photons and all showers not linked to charged particles are also shown for consistency checks. Only statistical errors are shown. 


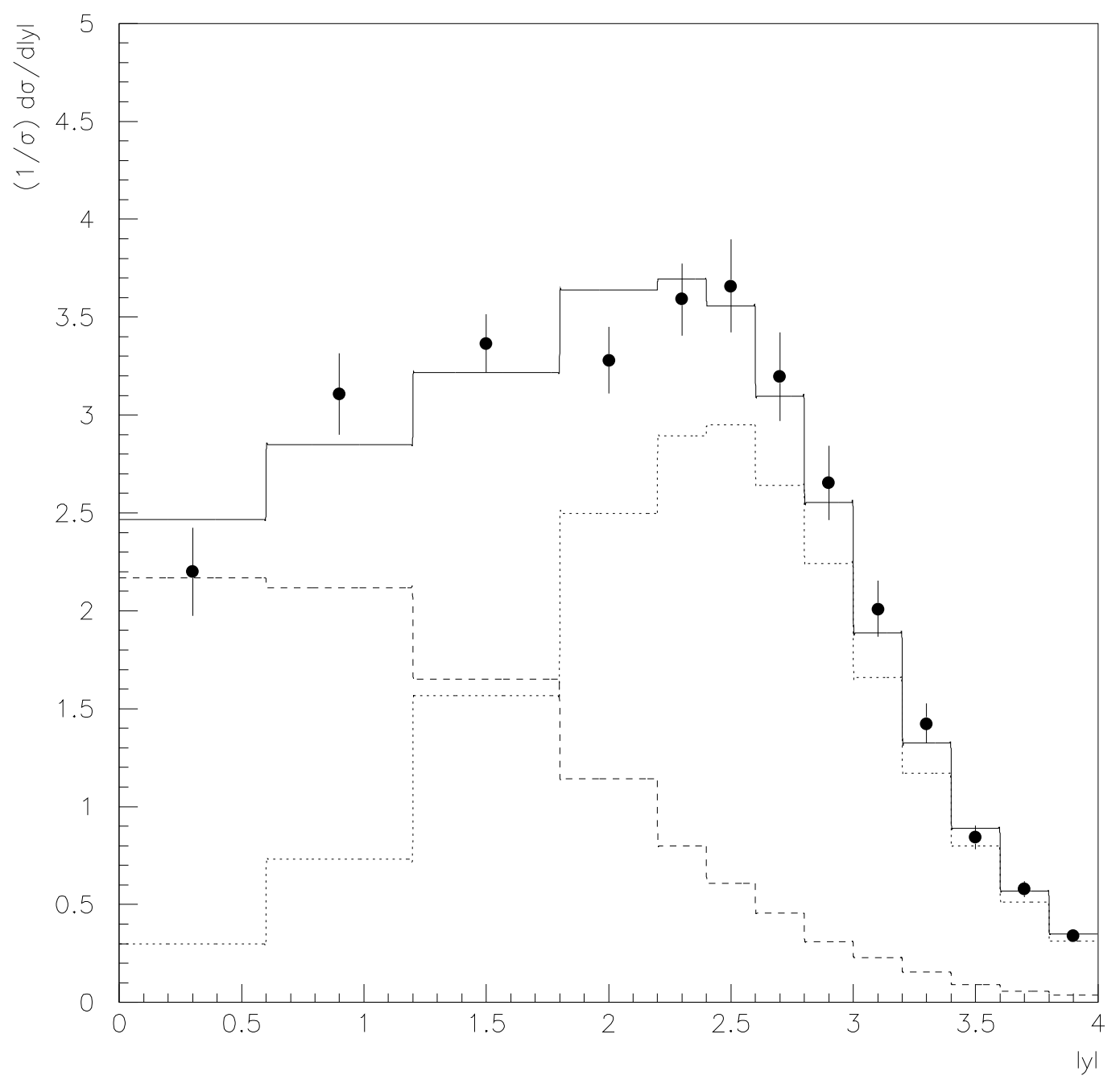

Figure 14: Rapidity distribution $r(|y|)$ (with respect to the thrust axis of the event) of $\pi^{0}$ in $b \bar{b}$ events. The two components from decay of $\mathrm{B}$ hadrons (dotted line) and fragmentation (dashed line) (as in JETSET 7.3 PS) are shown along with their sum (solid line). 